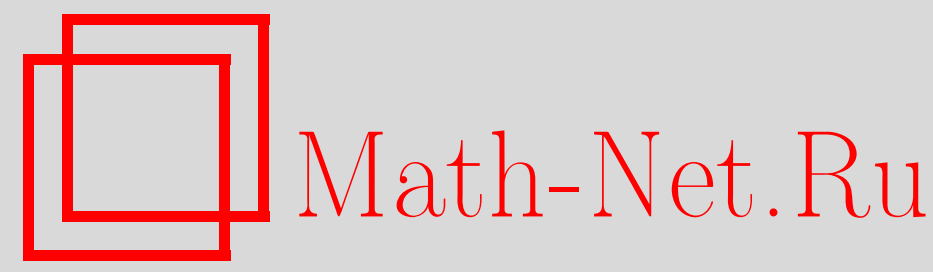

Е. П. Долженко, Е. А. Севастьянов, Аппроксимация со знакочувствительным весом (устойчивость, приложения к теории ужей и хаусдорфовым аппроксимациям), Изв. РАН. Сер. матем., 1999, том 63, выпуск 3, 77-118

DOI: https://doi.org/10.4213/im243

Использование Общероссийского математического портала Math-Net.Ru подразумевает, что вы прочитали и согласны с пользовательским соглашением

http://www . mathnet.ru/rus/agreement

Параметры загрузки:

IP: 54.237 .206 .68

26 апреля 2023 г., 14:42:13 
УДК 517.518 .8

\author{
Е. П. Долженко, Е. А. Севастьянов
}

\title{
Аппроксимация со знакочувствительным весом (устойчивость, приложения к теории ужей и хаусдорфовым аппроксимациям)
}

\begin{abstract}
Знакочувствительные аппроксимации учитывают не только модуль ошибки приближения, но и ее знак. В предыдущей работе с тем же названием и подзаголовком "теоремы существования и единственности" изучались вопросы существования, единственности и множественности элемента наилучшего равномерного приближения со знакочувствительным весом $p=\left(p_{-}, p_{+}\right)\left(p_{ \pm}(x) \geqslant 0, x \in E\right)$ некоторым (в частности, чебышевским) семейством $L$ ограниченных функций на множестве $E \subset \mathbb{R}$. Важную роль при этом играли понятия жесткости и свободы системы $(p, L)$. Здесь же мы рассмотрим вопрос об устойчивости такого процесса приближения, т.е. о непрерывной зависимости от $p$ наименьших уклонений $E(p, L, f)$ и наилучших приближений $l(p, L, f)$ функций $f$ элементами $l \in L$ при изменениях $p$, оцениваемых в так называемой $d$-метрике. Результаты прилагаются к теории ужей и хаусдорфовым аппроксимациям специальных многозначных функций.
\end{abstract}

Библиография: 12 наименований.

\section{Введение}

Ниже $C(E)$ - пространство всех ограниченных и непрерывных функций на множестве $E$ с нормой $\|g\|=\|g\|_{C(E)}:=\sup \{|g(x)|: x \in E\}, M(E)$ - пространство всех ограниченных функций на $E$ с той же нормой.

Пусть $P$ - неотрищательный сублинейный функционал на линейном нормированном пространстве $\mathscr{L}$ с нормой $\|\cdot\|$; если $L \subset \mathscr{L}$, то положим

$$
\begin{aligned}
\|P\|_{L} & :=\sup \{|P(l)| /\|l\|: l \in L, l \neq 0\}, \quad\|P\|:=\|P\|_{\mathscr{L}}, \\
W(P ; L) & =\sup \{\|f\| / P(f): f \in L, f \neq 0\}, \\
S(P ; L) & =\inf \{P(f) /\|f\|: f \in L, f \neq 0\}
\end{aligned}
$$

- свобода и жсесткость системы $(P ; L)(L \subset \mathscr{L})$. Очевидно, $0 \leqslant S(P ; L) \leqslant\|P\|_{L}$. Через $E(P ; L, f):=\inf \{P(l-f): l \in L\}, l(P ; L, f)$ и $A(P ; L, f)$ обозначим наименьшее уклонение $L$ от $f \in \mathscr{L}$, соответствуюший элемент наилучшего приближения (если он сушествует) и множество всех $l(P ; L, f)$ для $f ; E(L, f):=E(\|\cdot\| ; L, f)$.

Напомним, что чебьшевским подпространством на промежутке $\Delta=\langle a, b\rangle$ называется такое конечномерное (линейное) подпространство $L$ из $C(\Delta)$, что любая функция $l \in L$ имеет на $\Delta$ не более $\operatorname{dim} L-1$ различных нулей. Семейство всех $n$-мерных чебышевских подпространств на промежутке $\Delta$ будем обозначать $\mathrm{TCH}_{n}(\Delta)$.

(C) Е.П. ДолЖенко, Е.А. Севастьянов 1999 
Знакочувствительныл весом (ниже обозначаемым термином "вес") на множестве $E$ назовем пару $p=\left(p_{-}, p_{+}\right)$неотрицательных фуннкий $p_{-}(x), p_{+}(x)$ на $E$; вес $р$ называется ограниченным, непрерывным, полунепрерывным, если таковы и $p_{-}$, и $p_{+}$. Полунепрерывная сверху регуляризачия $\bar{g}(x)$ функции $g(x)(x \in E)$, т.е. ее верхняя функция Бэра, определяется на замыкании $\bar{E}$ множества $E$ равенством

$$
\bar{g}(t):=\varlimsup_{x \rightarrow t} g(x)=\lim _{s \rightarrow 0} \sup \{g(x): x \in E,|x-t|<s\}
$$

полунепрерывная сверху регуляризация веса $p=\left(p_{-}, p_{+}\right)-\sec \bar{p}=\left(\bar{p}_{-}, \bar{p}_{+}\right)$. Положим

$$
f^{+}(x):=\max \{f(x), 0\}, \quad f^{-}(x):=(-f(x))^{+}
$$

(очевидно, $f=f^{+}-f^{-},|f|=f^{+}+f^{-}$);

$$
(f, p)(x):=f^{+}(x) p_{+}(x)-f^{-}(x) p_{-}(x), \quad|f|_{p, E}:=\|(f, p)\|_{C(E)}
$$

$\|p\|:=\max \left\{\left\|p_{-}\right\|,\left\|p_{+}\right\|\right\}$(при $\|p\|=1$ вес $p$ назовем нормированнылм); $\operatorname{supp}(g)-$ замкнутый носитель функции $g(x)(x \in E \subset \mathbb{R})$, т.е. замыкание в $E$ множества $\{x \in E: g(x) \neq 0\} ; \operatorname{supp}(p):=\operatorname{supp}\left(p_{-}\right) \cup \operatorname{supp}\left(p_{+}\right)$.

Свобода $W(p, L)$ и жесткость $S(p, L)$ системы $(p, L)$ из веса $p$ на $E$ и множества $L \subset M(E)$, а также наименьшее уклонение $E(p, L, f)$, наилучшее приближсение $l(p, L, f)$ и множество $A(p, L, f)$ всех $l(p ; L, f)$ определяются как $W(P ; L)$ и $S(P ; L), E(P ; L ; f), l(P ; L ; f)$ и $A(P ; L ; f)$ при $P(\cdot)=|\cdot|_{p, E}(f \in M(E))$. Отметим, что для любого (не обязательно конечного) веса $p$ функционал $|\cdot|_{p, E}$ на пространстве $\mathscr{L}=M(E)$ полунепрерывен снизу [1, п. 0.1], если же вес $p$ ограничен, то этот функционал также ограничен; очевидно, $S(p, L) \leqslant\|p\|$.

Ниже нам понадобится условие конечности свободы системы $(p, L)$ в случае чебышевского подпространства $L$.

0.1. ТЕОРемА. Пусть $L-n$-мерное чебышевское подпространство на отрезке $\Delta, \quad p=\left(p_{-}, p_{+}\right)$- произвольный вес на $\Delta$ (не обязательно конечный). Тогда для конечности свободы $W(p, L)$ необходимо и достаточно выполнения одного из условий:

а) на $\Delta$ есть возрастающая последовательность из $n+1$ точек, в котоpых $p_{-}(x)$ и $p_{+}(x)$ попеременно положительны (начиная с любой из них);

b) на $\Delta$ имеется ровно п точек, в которьх и $p_{-}(x)$, и $p_{+}(x)$ положительньь, в остальных же точках обе они равны нулю (см. [1, следствие теоремы 2.7]; а также [2]).

Если $L \subset \mathscr{L}, \mathrm{P}$ - некоторое семейство неотрицательных сублинейных функционалов на $\mathscr{L}$, то свободой и жесткостью суперсистемы $(\mathrm{P} ; L)$ назовем величины

$$
W(\mathrm{P} ; L):=\sup \{\|l\| / P(l): l \in \mathscr{L}, l \neq 0, P \in \mathrm{P}\}
$$

и

$$
S(\mathrm{P} ; L):=\inf \{P(l) /\|l\|: l \in \mathscr{L}, l \neq 0, P \in \mathrm{P}\}
$$


соответственно. Если $\mathrm{p}$ - некоторое семейство знакочувствительных весов на множестве $E, L \subset M(E)$, то свободой и жесткостью суперсистемы $(\mathrm{p}, L)$ назовем соответственно $W(\mathrm{P} ; L)$ и $S(\mathrm{P} ; L)$ с $\mathrm{P}=\left\{|\cdot|_{p, E}: p \in \mathrm{p}\right\}$.

Напомним теорему о $p$-альтернансе (см. [1, теорема 3.6$]$, а также [3]).

Назовем семейство точек $x_{1}<x_{2}<\ldots<x_{n}$ из $E \subset \mathbb{R} n$-точечныцм $p$-альтернансом пары $f, g \in M(E)$ (на $E$ ), если с возрастанием целого $k$ от 1 до $n$ величина $(f-g, p)\left(x_{k}\right)$ с каждым шагом меняет знак, оставаясь по абсолютной величине равной $|f-g|_{p, E}$. Следующее утверждение (см. $[1$, п. 3.6], [3]) и по формулировке, и по доказательству - достаточно простое обобшение теоремы Чебышева об альтернансе.

0.2. ТеОрема (о $p$-альтернансе). Пусть $L-n$-мерное чебышевское подпространство на отрезке $\Delta(n \geqslant 1), \quad p=\left(p_{-}, p_{+}\right)$- конечный и полунепрерывный сверху вес на $\Delta, f \in C(\Delta), E(p, L, f)>0$. Тогда для того чтобы функиия $l \in L$ была әлементом наилучшего приближсения в $L$ для $f$ на $\Delta c$ весом р, необходимо и достаточно наличие у пары $l, f$ на $\Delta(n+1)$-точечного р-альтернанса.

Важную роль ниже играет так называемое $d$-расстояние (см. [1], [2], [4]). Перед его определением напомним некоторые известные понятия.

Расстояние Минковского $\rho_{M}$ на плоскости $x O y$ между точками $(x, y)$ и $(u, v)$ определяется равенством

$$
\rho_{M}((x, y),(u, v))=\max \{|x-u|,|y-v|\}
$$

Множество $F$ на плоскости $x O y$ называется выпуклым относительно оси $O y$, если вместе с любыми двумя точками из $F$, имеющими одну и ту же абсциссу, в $F$ входит соединяюший их вертикальный отрезок.

Дополненныц графиком $F(f)$ функции $y=f(x)$ (вообще говоря, неоднозначной) называется наименьшее замкнутое множество, выпуклое относительно направления оси $O y$ и содержащее график этой функции; дополненный график ограниченной функции, заданной на промежутке (т.е. на замкнутом или полузамкнутом отрезке, или на открытом интервале), является замкнутым связным множеством - континуумом, если промежуток конечен. Хаусдорфовым расстоянием $h(A, B)$ между двумя ограниченными множествами $A$ и $B$ на плоскости $x O y$ называется инфимум всех таких $r>0$, что $A$ и $B$ содержатся друг у друга в $r$-окрестностях Минковского; хаусдорфовым расстоянием между двумя ограниченны.ми функиия.ми $g$ и $h$, определенными на некотором множестве $E \subset \mathbb{R}$, называется $H(g, h):=h(F(g), F(h))$.

ОПРЕДЕЛЕНИЕ. $d$-расстоянием между функциями $g, h \in M(E)$ назовем инфимум $d(g, h)$ всех чисел $r>0$, при которых график функции $M(g, h ; x):=$ $\max \{g(x), h(x)\}, x \in E$, содержится в $r$-окрестности Минковского каждого из их дополненных графиков $F(g)$ и $F(h) ; d$-расстоянием междду ограниченными на $E \subset \mathbb{R}$ весами $p=\left(p_{-}, p_{+}\right)$и $q=\left(q_{-}, q_{+}\right)$назовем $d(p, q):=\max \left\{d\left(p_{-}, q_{-}\right)\right.$, $\left.d\left(p_{+}, q_{+}\right)\right\}$. 
При $f, g \in M(E)$ имеем для $d$-расстояния, хаусдорфова $H(f, g)$ и равномерного $\|f-g\|$ расстояний

$$
d(f, g) \leqslant H(f, g) \leqslant\|f-g\|
$$

и если $E-$ промежуток, то и равенство

$$
H(f, g)=\max \{d(f, g), d(-f,-g)\}
$$

если $f, g \in M(E), f, g \geqslant 0$, веса $p, q$ ограничены, то

$$
|\|f\|-\|g\|| \leqslant d(f, g), \quad|\|p\|-\|q\|| \leqslant d(p, q)
$$

Семейство всех ограниченных полунепрерывных сверху весов $p=\left(p_{-}, p_{+}\right)$на множестве $E \subset \mathbb{R}$ с метрикой $d(p, q)$ обозначим $\operatorname{DS}(E)$. Отметим (cм. [1], [2], [4]), что если $\Delta$ - промежуток на $\mathbb{R}$, то $\operatorname{DS}(\Delta)$ является полным метрическим пространством, сепарабельным при конечном $\Delta$ и несепарабельным в противном случае.

Для сокращения записи положим $t p=\left(t p_{-}, t p_{+}\right)$при $t=$ const $\in[0, \infty)$, $\tilde{f}:=f /\|f\|(f \in M(E)), \tilde{p}:=p /\|p\|, D(p, q)=d(\tilde{p}, \tilde{q})$. Наряду с $d(p, q), D(p, q)$ используется ниже как мера взаимного отклонения нетривиальных (т.е. отличных от веса $0:=(0,0))$ знакочувствительных весов $p, q$. Сфера $S(\Delta)=\{p \in$ $\mathrm{DS}(E): d(p, 0)=1\}$ является полным метрическим пространством с метрикой $D(p, q)=d(p, q)$. Если в $\operatorname{DS}(\Delta) \backslash\{0\}$ ввести соотношение эквивалентности $p \sim q \Leftrightarrow \tilde{p}=\tilde{q}$, то получим полное метрическое пространство $\mathrm{DS}^{+}(\Delta)$ с метрикой $D(p, q)$. На множестве всех ограниченных весов $p, q$ (на $E \subset \mathbb{R}) d(p, q)$ не является метрикой, однако мы будем ее использовать для оценки взаимного отклонения весов $p, q$ и в этом случае.

В радиотехнике обобщенной расстройкой частот $f, \varphi$ называется величина

$$
k(\varphi, f):=\frac{1}{2}\left|\frac{\varphi}{f}-\frac{f}{\varphi}\right| .
$$

Если $P$ и $Q$ - конечные неотрицательные функционалы на некотором подмножестве $L$ линейного нормированного пространства $\mathscr{L}$, то обобщенной расстройкой $P$ и $Q$ на $L$ назовем число

$$
\begin{aligned}
k(P ; Q ; L) & :=\sup \{k(P(l), Q(l)): l \in L\}, \\
k(0,0) & :=0, \quad k(0, a)=k(a, 0):=\infty \quad \forall a>0 .
\end{aligned}
$$

Если $f, g$ - определенные на $E$ конечные неотрицательные функции, $p, q$ - конечные веса на $E$, то

$$
\begin{aligned}
k(f, g) & =k(f, g ; E):=\|k(f(\cdot), g(\cdot))\|_{C(E)}, \\
k(p, q) & :=k(p, q ; E):=\max \left\{k\left(p_{-}, q_{-} ; E\right), k\left(p_{+}, q_{+} ; E\right)\right\}
\end{aligned}
$$


назовем обобщенными расстройками функций $f, g$ и весов $p, q$ соответственно. Очевидно,

$$
\begin{aligned}
& k(P ; Q ; L)=k(Q ; P ; L) \geqslant 0, \quad k(p, q)=k(q, p) \geqslant 0, \\
& k(P ; Q ; L)=0 \Leftrightarrow P(l)=Q(l) \quad \forall l \in L .
\end{aligned}
$$

При $E \subset \mathbb{R}, L \subset M(E), f \in M(E), p, q \not \equiv(0,0)$ и $t \geqslant 0$ положим

$$
\begin{gathered}
L(f):=\{l-f: l \in L\}, \quad \operatorname{dif} L:=\{f-g: f, g \in L\}, \\
W(p, q, L):=\max \{W(p, L), W(q, L)\} .
\end{gathered}
$$

Отметим, что $W(P, L(f))$ оценивается более простой величиной $W(P, \operatorname{dif} L)$ (см. [1, теорема 2.13]).

0.3. ТеОрема. Если $P$ - ограниченный сублинейный функчионал на линейном нормированном пространстве $\mathscr{L}, L \subset \mathscr{L}, f \in \mathscr{L}, E(P ; L, f)>0$, mo

$$
W(P ; L(f)) \leqslant 3\|P\| \frac{E(\|\cdot\| ; L, f)}{E(P ; L, f)} \cdot W(P ; \operatorname{dif} L) .
$$

Заметим, что если $P$ - полунепрерывный снизу сублинейньй функционал на линейном нормированном пространстве $\mathscr{L}, L$ - (линейное) конечномерное подпространство в $\mathscr{L}, f \in \mathscr{L}$ и $W(P ; L(f))<\infty$, то $f$ имеет хотя бы один элемент наилучшего приближения $l(P ; L ; f)$; если условие $W(P ; L(f))<\infty$ здесь заменить условием $W(P ; L)<\infty$, то каждый элемент $f \in \mathscr{L}$ имеет $l(P ; L ; f)$. Действительно, если $f \in \mathscr{L}, l_{j} \in L, P\left(l_{j}-f\right) \rightarrow E(P ; L ; f)$, то при $W(P ; L(f))<\infty$ имеем

$$
\left\|l_{j}\right\| \leqslant\left\|l_{j}-f\right\|+\|f\| \leqslant W(P ; L(f)) P\left(l_{j}-f\right)+\|f\| \leqslant C,
$$

а при $W(P ; L)<\infty$

$$
\left\|l_{j}\right\| \leqslant W(P ; L) P\left(l_{j}\right) \leqslant W(P ; L) P\left(l_{j}-f\right)+W(P ; L) P(f) \leqslant C
$$

$(C$ не зависит от $j)$. Так как последовательность $\left\{l_{j}\right\}$ имеет на $L$ хотя бы одну предельную точку $\bar{l}, P$ полунепрерьвен снизу, то $P(\bar{l}-f)=E(P ; L ; f)$.

Ниже также используются обозначения

$\omega(f, t)=\omega(f, E, t):=\sup \left\{\left|f\left(x^{\prime}\right)-f\left(x^{\prime \prime}\right)\right|: x^{\prime}, x^{\prime \prime} \in E,\left|x^{\prime}-x^{\prime \prime}\right| \leqslant t\right\}$, $\omega(L, t)=\omega(L, E, t):=\sup \{\omega(l /\|l\|, E, t): l \in L, l(x) \not \equiv 0\}, \quad L \subset M(E), \quad t \geqslant 0$ $(\omega(f, t)$ и $\omega(L, t)$ - модули непрерывности функции $f$ и семейства $L)$,

$$
\sigma(p, q, L ; t):=\max \{\|p\|,\|q\|\} \cdot \omega(L, t+0)+t
$$

$(p, q$ - знакочувствительные веса на $E \subset \mathbb{R}, L \subset M(E))$.

Ниже будет неоднократно использоваться следующая теорема об устойчивости жесткости и свободы системы $(p, L)$ (см. [1, п. 2.14], [2]).

0.4. Теорема. Если $L \subset M(E)(E \subset \mathbb{R})$, веса $p, q$ ограничень, то

$$
|S(q, L)-S(p, L)| \leqslant \underline{\sigma}:=\min \{\|p\|,\|q\|\} \cdot \omega(L, d+0)+d, \quad d=d(p, q) .
$$

Если при этом $\underline{\sigma}<s:=S(p, L)$, mo

$$
\frac{W(p, L)}{1+\underline{\sigma} W(p, L)} \leqslant W(q, L) \leqslant \frac{W(p, L)}{1-\underline{\sigma} W(p, L)}=\frac{1}{s-\underline{\sigma}} .
$$




\section{ГлАВА 1. Устойчивость}

\section{§ 1. Устойчивость наименьших уклонений}

1.0. В этом параграфе рассматривается вопрос об устойчивости величин $E(p, L, f)$ и $E(P ; L, f)$ при вариациях знакочувствительного веса $p$ и сублинейного функционала $P$ соответственно.

Следуюшие леммы ниже используются неоднократно.

1.1. Лемма. Если веса $p, q$ ограничены на $E, d=d(p, q), L \subset M(E), m o$ для сублинейных функиионалов $P=|\cdot|_{p, E}, Q=|\cdot|_{q, E}$ имеем при $l \in L$

$$
\begin{gathered}
Q(l) \leqslant P(l)+\|q\| \omega(l, d+0)+\|l\| d \\
\leqslant P(l)+\|l\| \cdot(\|q\| \omega(L, d+0)+d), \\
|P(l)-Q(l)| \leqslant \max \{\|p\|,\|q\|\} \omega(l, d+0)+\|l\| d \leqslant\|l\| \cdot \sigma(p, q, L ; d), \\
P(l) \leqslant Q(l)+Q(l) \cdot y, \quad y=W(q, L)(\|p\| \cdot \omega(L, d+0)+d), \\
Q(l) \leqslant P(l)+P(l) \cdot z, \quad z=W(p, L)(\|q\| \cdot \omega(L, d+0)+d), \\
k(P ; Q ; L) \leqslant \max \{\|q\| W(p, L),\|p\| W(q, L)\} \cdot \omega(L, d+0) \\
\quad+\max \{W(p, L), W(q, L)\} d \leqslant W(p, q, L) \cdot \sigma(p, q, L ; d) .
\end{gathered}
$$

ДокАЗАТЕЛЬСТвО. Пусть $\varepsilon>0, l \in L$. По определению $d(p, q)$ для каждого $x \in E$ найдется такое $t \in E$, что $|x-t|<d+\varepsilon, q_{+}(x)<p_{+}(t)+d+\varepsilon$. Поэтому

$$
\begin{aligned}
l(x)^{+} q_{+}(x)= & (l(t)+l(x)-l(t))^{+} q_{+}(x) \leqslant l(t)^{+} p_{+}(t)+l(t)^{+}(d+\varepsilon) \\
& +(l(x)-l(t))^{+} q_{+}(x) \leqslant P(l)+\|l\|(d+\varepsilon)+\|q\| \omega(l, d+\varepsilon) .
\end{aligned}
$$

Очевидно, эти неравенства останутся в силе, если в них индексы + всюду заменить индексами -. Поскольку при каждом $a \in \mathbb{R}$ лишь одна из двух величин $a^{-}, a^{+}$ может быть ненулевой, то из этих неравенств при $x \in E$ имеем

$$
\begin{aligned}
|(l, q)(x)| & \leqslant P(l)+\|l\|(d+\varepsilon)+\|q\| \omega(l, d+0), \\
Q(l) & \leqslant P(l)+\|q\| \omega(l, d+0)+\|l\| d \\
& \leqslant P(l)+\|l\| \cdot(\|q\| \omega(L, d+0)+d),
\end{aligned}
$$

т.е. (1.1.0). Так как здесь $p$ и $q$ можно менять местами, то доказано (1.1.1).

Далее, $\|l\| \leqslant Q(l) W(q, L),\|l\| \leqslant P(l) W(p, L)$, так что

$$
\begin{aligned}
& P(l) \leqslant Q(l)+Q(l) W(q, L)(\|p\| \omega(L, d+0)+d), \\
& Q(l) \leqslant P(l)+P(l) W(p, L)(\|q\| \omega(L, d+0)+d),
\end{aligned}
$$

и (1.1.2) доказано. 
Поскольку $k(a, b)=k(b, a)=(|a-b| / b)\{(a+b) /(2 a)\}$, при $a=P(l) \geqslant Q(l)=b$ из (1.1.5) имеем

$$
k(P(l), Q(l)) \leqslant W(q, L)(\|p\| \omega(L, d+0)+d),
$$

а при $a=Q(l) \geqslant P(l)=b$

$$
k(P(l), Q(l)) \leqslant W(p, L)(\|q\| \omega(L, d+0)+d)
$$

(уже из (1.1.6)). Это дает (1.1.3).

1.2. ЛЕмма. Для неотрицательного функиионала $F(x)$, определенного и ограниченного на некотором множестве $X$, nусть $m(F)$ и $M(F)$ обозначают соответственно его инфимум и супремум на $X$. Тогда для любой пары $A, B$ таких функционалов имеем неравенства

$$
\begin{aligned}
& \max \{k(m(A), m(B)), k(M(A), M(B))\} \leqslant k(A ; B ; X), \\
& \max \{|m(A)-m(B)|,|M(A)-M(B)|\} \leqslant \sup \{|A(x)-B(x)|: x \in X\} .
\end{aligned}
$$

ДокАЗАТЕЛЬСтво. Если, например, $M(A)>M(B)$, то при $0<\varepsilon<$ $M(A)-M(B)$ и некотором $t \in X$ имеем

$$
\begin{gathered}
A(t)>M(A)-\varepsilon>M(B) \geqslant B(t), \\
k(M(A)-\varepsilon, M(B)) \leqslant k(A(t), M(B)) \leqslant k(A(t), B(t)), \quad k(M(A), M(B)) \leqslant C,
\end{gathered}
$$

где $C$ обозначает правую часть неравенства (1.2.1). Аналогично доказывается неравенство $k(m(A), m(B)) \leqslant C$ и неравенство (1.2.2).

1.3. ТеОрема. Если веса $р$ и q ограничены на $E \subset \mathbb{R}, \bar{p}$ и $\bar{q}$ - их полунепрерыьвнье сверху регуляризации, $d=d(p, q), L-$ множество из $M(E)$, то для сублинейных функиионалов $P=|\cdot|_{p, E} u Q=|\cdot|_{q, E}, \quad \bar{P}=|\cdot|_{\bar{p}, \bar{E}} u \bar{Q}=|\cdot|_{\bar{q}, \bar{E}}$ имеем неравенства

$$
\begin{aligned}
& \|P-Q\|_{L} \leqslant \sigma(p, q, L ; d), \\
& k(P ; Q ; L) \leqslant W(p, q, L) \cdot \sigma(p, q, L ; d), \\
& k(P ; Q ; L) \leqslant k(P ; Q ; M(E))=k(p, q) \quad n p u \quad L \subset M(E) \text {, } \\
& k(\bar{P} ; \bar{Q} ; L) \leqslant k(\bar{P} ; \bar{Q} ; C(\bar{E}))=k(\bar{p}, \bar{q}) \quad \text { npu } \quad L \subset C(\bar{E}) .
\end{aligned}
$$


ДокАЗАТЕЛЬСтво. Неравенство (1.3.1) немедленно следует из (1.1.1), (1.3.2)это (1.1.3). Полагая, как обычно, $\|\cdot\|:=\|\cdot\|_{E}$, из леммы 1.2 при $X=E$, $A(x)=l^{-}(x) p_{-}(x)$ и $B(x)=l^{-}(x) q_{-}(x)$ получаем

$$
\begin{aligned}
k\left(\left\|l^{-} p_{-}\right\|,\left\|l^{-} q_{-}\right\|\right) & \leqslant\left\|k\left(l^{-}(\cdot) p_{-}(\cdot), l^{-}(\cdot) q_{-}(\cdot)\right)\right\| \\
& \leqslant\left\|k\left(p_{-}(\cdot), q_{-}(\cdot)\right)\right\| \leqslant k(p, q), \quad l \in L,
\end{aligned}
$$

и аналогичное неравенство с заменой индексов - индексами + (знак $<$ в неравенстве $k(t a, t b) \leqslant k(a, b)$ появляется при $t=0, a \neq b)$. Поэтому для тех $l \in L$, для которых $P(l)=\left\|l^{-} p_{-}\right\|$и $Q(l)=\left\|l^{-} q_{-}\right\|$или $P(l)=\left\|l^{+} p_{+}\right\|$и $Q(l)=\left\|l^{+} q_{+}\right\|$, имеем неравенство

$$
k(P(l), Q(l)) \leqslant k(p, q) .
$$

Если же для $l \in L$ ни одна из этих пар равенств не выполнена, например, если $P(l)=\left\|l^{-} p_{-}\right\|, \quad Q(l)=\left\|l^{+} q_{+}\right\|$и $P(l)>Q(l) \quad\left(>\left\|l^{-} q_{-}\right\|\right)$, то из определения обобшенной расстройки (см. введение) получим неравенство $k(P(l), Q(l)) \leqslant$ $k\left(\left\|l^{-} p_{-}\right\|,\left\|l^{-} q_{-}\right\|\right)$, а из него и из (1.3.5) - опять неравенство (1.3.6). Таким обра30M,

$$
k(P ; Q ; L) \leqslant k(P ; Q ; M(E)) \leqslant k(p, q) .
$$

Пусть, далее, $\varepsilon>0$, и при $t \in E$ положено $l_{t}(t)=-1, l_{t}(x)=0$ при $x \neq t$. Тогда если $k(p, q)=k\left(p_{-}, q_{-}\right)$(случай $k(p, q)=k\left(p_{+}, q_{+}\right)$исчерпывается аналогично с заменой $-l_{t}$ на $\left.l_{t}\right)$ и, например, $\left\|p_{-}\right\| \geqslant\left\|q_{-}\right\|$, то возьмем точку $t \in E$ так, чтобы было $k(p, q)-\varepsilon<k\left(p_{-}(t), q_{-}(t)\right)$. Тогда

$$
k(p, q)-\varepsilon<k\left(\left|l_{t}\right|_{p, E},\left|l_{t}\right|_{q, E}\right) \leqslant k(P ; Q ; M(E)),
$$

что ввиду произвольности $\varepsilon>0$ вместе с (1.3.7) дает (1.3.3).

Так как (1.3.3) доказано, то в (1.3.4) осталось доказать лишь равенство $k(P ; Q ; C(E))=k(p, q)$ для случая полунепрерывных сверху весов $p$ и $q$ на замкнутом множестве $E$. Для этого надо просто повторить рассуждения предыдущего абзаца, взяв в качестве $l_{t}$ непрерьвную неположительную функцию, равную -1 при $x=t$ и столь быстро возрастающую к 0 при удалении $x$ от $t$, что

$$
|l|_{p, E}=l_{t}^{-}(t) p_{-}(t)=p_{-}(t)
$$

(она существует ввиду полунепрерывности $p_{-}$сверху). Теорема доказана.

То, что в теореме $1.3 p$ и $q$ входят симметрично, является одновременно ее и достоинством, и недостатком (взгляните на правые части неравенств (1.3.1) и (1.3.2)). В следующем утверждении, вытекающем из (1.3.2) и (0.4.2), опорным является вес $p$.

1.4. СлЕДСТвИЕ. Если р и $q$-ограниченные веса на $E \subset \mathbb{R}, \quad P=|\cdot|_{p, E}$, $Q=|\cdot|_{q, E}, \quad d=d(p, q), \quad \sigma=\sigma(p, q, L ; d)<s:=S(p, L), m o$

$$
k(P ; Q ; L) \leqslant \frac{\sigma}{s-\sigma} .
$$


1.5. Теорема. Пусть $E \subset \mathbb{R}, L \subset M(E), \quad f \in M(E)$, веса $p$ и q на $E$ ограничены, $d=d(p, q)$. Тогда если $W(p, L(f))<\infty u W(q, L(f))<\infty$, то

$$
\begin{aligned}
& |E(p, L, f)-E(q, L, f)| \\
& \quad \leqslant \max \{E(p, L, f) W(p, L(f)), E(q, L, f) W(q, L(f))\} \cdot \sigma(p, q, L(f), d),(1.5 .1) \\
& k(E(p, L, f), E(q, L, f)) \leqslant W(p, q, L(f)) \cdot \sigma(p, q, L(f) ; d) .
\end{aligned}
$$

ДокАЗАТЕЛЬСТво. Из (1.1.2) получаем неравенства

$$
E(p, L, f) \leqslant E(q, L, f)(1+y), \quad E(q, L, f) \leqslant E(p, L, f)(1+z),
$$

что и дает (1.5.1). При $X=L(f), A=P=|\cdot|_{p, E}$ и $B=Q=|\cdot|_{q, E}$ из (1.2.1) и (1.1.3) вытекает (1.5.2).

Отметим, что в случае замкнутости $E$ при $f \in C(E), L \subset C(E)$ можно справа в неравенствах (1.5.1) и (1.5.2) вместо $p$ и $q$ писать их регуляризации $\bar{p}$ и $\bar{q}$.

Для пользования неравенством (1.5.2) надо, кроме $d(p, q)$, знать хотя бы оценки сверху для $W(p, L(f))$ и $W(q, L(f))$, а для использования (1.5.1) еще и оценки для $E(p, L, f)$ и $E(q, L, f)$. Это - плата за симметрию этих неравенств относительно $p$ и $q$. Однако, как и при доказательстве 1.4 , мы с помощью теоремы 0.4 можем в (1.5.2) сделать опорным вес $p$. Замечая, что $\|q\| \leqslant\|p\|+d(p, q)$, получаем

1.6. СледСтвиЕ. Если $E \subset \mathbb{R}, L \subset M(E), f \in M(E)$, веса $p$ и q на $E$ ограниченьь, $d=d(p, q), \quad \sigma_{f}:=(\|p\|+d) \omega(L(f), d+0)+d<s_{f}=S(p, L(f))$, mo

$$
k(E(p, L, f), E(q, L, f)) \leqslant \frac{\sigma_{f}}{s_{f}-\sigma_{f}} .
$$

Приведем еще одно неравенство, в котором $W(p, L(f))$ и $W(q, L(f))$ заменены величинами, уже не зависящими от $f$ и совпадаюшими соответственно с $W(p, L)$ и $W(q, L)$ в случае линейного подпространства $L$. Обратим внимание на то, что при этом можно оценить лишь разность между величинами $E(p, L, f)$ и $E(q, L, f)$, но, вообще говоря, нельзя оценить их относительную или обобщенную расстройки. Действительно, пусть, например, $L$ - множество алгебраических полиномов степени $\leqslant n(n \geqslant 1), E=\Delta=[-1,1] ; p(x) \equiv(1,1), 0<t=$ const $<1$, $q(x)=(1,1)$ при $t \leqslant|x| \leqslant 1$ и $q(x)=(t, t)$ при $|x|<t ; f(x)=0$ при $t \leqslant|x| \leqslant 1$, $f(x)=t \sin (2 \pi n x / t)$ при $|x|<t$. Легко видеть, что $\|p\|=\|q\|=1, d(p, q)=t / 2$ при $t<2 / 3, W(p, L)=1, W(q, L) \rightarrow 1(t \rightarrow 0), E(p, L, f)=t, E(q, L, f)=t^{2}$, $|E(p, L, f)-E(q, L, f)| / E(q, L, f)=1-t \rightarrow 1, k(E(p, L, f), E(q, L, f)) \rightarrow \infty$ $(t \rightarrow 0)$. Отметим, что здесь $f \in C(\Delta), p$ и $q$ полунепрерывны сверху, $L-$ конечномерное подпространство в $C(\Delta)$.

В соответствии с принятыми обозначениями ниже $E(L, f)$ обозначает наименьшее уклонение $L$ от $f$ в равномерной метрике: $E(L, f)=E\left(p_{0}, L, f\right)$ при $p_{0} \equiv(1,1)$. 
1.7. СлЕДСтвИЕ. Если $E \subset \mathbb{R}, L \subset M(E)$, веса $р$ и q ограничены на $E$, $d=d(p, q), \quad W:=\max \{W(p /\|p\|, \operatorname{dif} L), W(q /\|q\|, \operatorname{dif} L)\}, \quad E(p, L, f)>0 u$ $E(q, L, f)>0, m o$

$|E(p, L, f)-E(q, L, f)| \leqslant 3 W E(L, f)(\max \{\|p\|,\|q\|\} \cdot \omega(L(f), d+0)+d) .(1.7 .1)$

Если $E$ - компакт, $L \subset C(E), \quad f \in C(E)$, то условия $E(p, L, f)>0$, $E(q, L, f)>0$ не нужны.

ЗАмечаниЕ. В [1, лемма 2.10] приводится оценка для $\omega(L(f), \delta)$ : если $L \subset M(E), f \in M(E), E(L, f)>0, \varepsilon>0, l \in L,\|l-f\| \leqslant E(L, f)+\varepsilon$, то

$$
\frac{\omega(l-f, \delta)}{E(L, f)+\varepsilon} \leqslant \omega(L(f), \delta) \leqslant \frac{\omega(l-f, \delta)}{E(L, f)}+\left(2+\frac{\varepsilon}{E(L, f)}\right) \omega(L, \delta) \quad \forall \delta>0 .
$$

Справедливость (1.7.1) при $E(p, L, f)>0$ и $E(q, L, f)>0$ вытекает из (1.5.1) и (0.3.1) (неравенства $W(p, L(f))<\infty$ и $W(q, L(f))<\infty$ следуют из определения $W(p, L(f)))$. Далее, случай $E(p, L, f)=E(q, L, f)=0$ (имеюший место, в частности, при $f \in L)$ и случай $W=\infty$ тривиальны (см. (1.7.1)). Если $W<\infty$ и, например, $E(p, L, f)=0, E(q, L, f)>0$, то (1.7.1) верно при замене $p$ на $p_{r}:=\left(p_{-}+r, p_{+}+r\right)$ с $r=$ const $>0$, a $W-$ на соответствуюшую величину $W_{r}$. Так как $\left\|p_{r}\right\|=\|p\|+r, d\left(p, p_{r}\right) \leqslant r$, то $W\left(p_{r}, L\right) \rightarrow W(p, L)$ при $r \rightarrow 0$ (см. (0.4.2)), и осталось сослаться на следующее утверждение.

1.7а. Лемма. Если $E$ - компакт, вес р ограничен, $L \subset C(E), f \in C(E)$, $E(p, L, f)=0, \quad p_{r}=\left(p_{-}+r, p_{+}+r\right)(r=$ const $>0), \operatorname{mo} E\left(p_{r}, L, f\right) \rightarrow 0$ npu $r \rightarrow 0$.

Это утверждение вытекает из неравенства (1.1.1), в котором положено $l=l_{\varepsilon}-f$, где $l_{\varepsilon} \in L$ и $P\left(l_{\varepsilon}-f\right)<\varepsilon, q=p_{r}$ и $P_{r}=Q\left(\right.$ в (1.1.1) считаем $\left.L=\left\{l_{\varepsilon}-f\right\}\right)$ :

$$
\left|P\left(l_{\varepsilon}-f\right)-P_{r}\left(l_{\varepsilon}-f\right)\right| \leqslant(\|p\|+r) \omega\left(l_{\varepsilon}-f, r\right)+\varepsilon r \rightarrow 0
$$

при $r \rightarrow 0$, откуда $E\left(p_{r}, L, f\right)<2 \varepsilon$ при $r<r(\varepsilon)$.

1.8. ТеоремА. Если веса $p$ и q ограничены на $E \subset \mathbb{R}, L \subset M(E), f \in M(E)$, $E(p, L, f)>0 u E(q, L, f)>0, m o$

$$
\begin{aligned}
& |E(p, L, f)-E(q, L, f)| \leqslant 3 W E(L, f)(N \omega(l, d+0)+d) \\
& \quad+N E(L, f) \omega(L, d+0)+N \omega(f, d+0)
\end{aligned}
$$

əде $d=d(p, q), W=\max \{W(p /\|p\|, \operatorname{dif} L), W(q /\|q\|, \operatorname{dif} L)\}, N=\max \{\|p\|,\|q\|\}$. Если $E$ - компакт, $L \subset C(E), \quad f \in C(E)$, то условия $E(p, L, f)>0$, $E(q, L, f)>0$ не нужны. 
ДокАЗАТЕЛЬСТво. Пусть сперва $E(p, L, f)>0, E(q, L, f)>0$. Из (1.1.0) при $\|\cdot\|=\|\cdot\|_{C(E)}, P(\cdot)=|\cdot|_{p, E}, Q(\cdot)=|\cdot|_{q, E}$ и $l \in L$ получаем

$$
\begin{aligned}
E(q, L, f) \leqslant & Q(l-f) \leqslant P(l-f)+\|q\| \omega(l-f, d+0)+\|l-f\| d \\
\leqslant & P(l-f)+\|l\|\|q\| \omega(L, d+0) \\
& +\|q\| \omega(f, d+0)+\|l-f\| d .
\end{aligned}
$$

Возьмем $\varepsilon>0$ и такой элемент $l \in L$, что $P(l-f)<E(p, L, f)+\varepsilon$. При $u=E(L, f)$ и $h=E(p, L, f)$ из (0.3.1) и определения $W(p, L(f))$ имеем неравенства

$$
\begin{gathered}
\|l-f\| \leqslant W(p, L(f)) P(l-f) \leqslant 3\|p\| W(p, \operatorname{dif} L)(u / h)(h+\varepsilon) \\
\|l\| \leqslant\|l-f\|+\|f\| \leqslant 3\|p\| W(p, \operatorname{dif} L)(1+\varepsilon / h) u+\|f\| .
\end{gathered}
$$

Из них и из (1.8.2) при $\varepsilon \rightarrow 0$ получаем соотношение

$$
\begin{aligned}
E(q, L, f)-E(p, L, f) \leqslant & \|q\|(3\|p\| W(p, \operatorname{dif} L) u+\|f\|) \omega(L, d+0) \\
& +\|q\| \omega(f, d+0)+3\|p\| W(p, \operatorname{dif} L) u d \\
= & 3 W(p /\|p\|, \operatorname{dif} L) u(\|q\| \omega(L, d+0)+d) \\
& +\|q\| \omega(f, d+0)+\|f\| \cdot\|q\| \omega(L, d+0)
\end{aligned}
$$

и аналогичное соотношение, в котором $p$ и $q$ меняются местами. Из этих двух неравенств и следует (1.8.1) с заменой второго множителя $E(L, f)$ на $\|f\|$. Отсюда, заменяя $f$ на $f-l_{r}$, где $\left\|l_{r}-f\right\|<E(L, f)+r\left(l_{r} \in L, r>0\right)$, при $r \rightarrow 0$ получаем (1.8.1).

Вторая часть теоремы доказывается так же, как вторая часть следствия 1.7 ссылкой на лемму 1.7а.

Учитывая, что $E(L, f) \leqslant\|f\|$ при $0 \in L$ и что $W(p, L) \geqslant 1$ при $\|p\|=1$, из теорем 1.8 и 0.4 получаем

1.9. СлЕДСТвИЕ. Если L-линейное подпространство в $C(E), f \in C(E)$, $\|p\|=\|q\|=1, \quad d=d(p, q), \quad \delta:=\omega(L, d+0)+d<S:=S(p, L), m o$

$$
|E(p, L, f)-E(q, L, f)| \leqslant 4\|f\| \cdot \frac{\delta}{S-\delta}+\omega(f, d+0)
$$

Из (1.2.1) и (1.3.3) при $X=L(f), A=P=|\cdot|_{p, E}$ и $B=Q=|\cdot|_{q, E}$ вытекает

1.10. Теорема. Если веса р и q ограничены на $E, L \subset M(E), f \in M(E)$, mo

$$
k(E(p, L, f), E(q, L, f)) \leqslant k(p, q) .
$$


1.11. ЗАмЕЧАнИЕ. Если в пп. $1.5-1.10$ считать веса $p, q$ нормированными, то соответствуюшие неравенства и их доказательства несколько упростятся. Из этих упрощенных неравенств, пользуясь легко проверяемыми неравенствами

$$
\begin{aligned}
& k(\lambda, \varkappa) \leqslant k\left(\frac{\lambda}{\|p\|}, \frac{\varkappa}{\|q\|}\right)(1+4 k(\|p\|,\|q\|))+k(\|p\|,\|q\|), \\
& |\lambda-\varkappa| \leqslant \max \{\|p\|,\|q\|\}\left|\frac{\lambda}{\|p\|}-\frac{\varkappa}{\|q\|}\right|+\max \left\{\frac{\lambda}{\|p\|}, \frac{\varkappa}{\|q\|}\right\}|\|p\|-\|q\||
\end{aligned}
$$

(справедливыми для любых положительных чисел $\lambda, \varkappa,\|p\|,\|q\|)$, можно снова получить соответствуюшие, уже видоизмененные неравенства для произвольных ограниченных нетривиальных весов $p, q$. Например, неравенство (1.6.1), заново доказанное для нормированных весов $\tilde{p}=p /\|p\|$ и $\tilde{q}=q /\|q\|$, имеет вид

$$
k(E(\tilde{p}, L, f), E(\tilde{q}, L, f)) \leqslant \frac{\delta_{f}}{S_{f}-\delta_{f}},
$$

где $\delta_{f}:=\omega(L(f), D+0)+D<S_{f}:=S(\tilde{p}, L(f)), D=D(p, q)=d(\tilde{p}, \tilde{q})$. Отсюда и из (1.11.1) при $\lambda=E(p, L, f)$ и $\varkappa=E(q, L, f)$ получим неравенство

$$
k(E(p, L, f), E(q, L, f)) \leqslant(1+4 k) \frac{\delta_{f}}{S_{f}-\delta_{f}}+k, \quad k=k(\|p\|,\|q\|) .
$$

Заметим, что $d=d(p, q)$ и $D=D(p, q)$ при фиксированном $p \not \equiv 0$ стремятся к нулю одновременно, $k \leqslant d /\|p\|+o(d)$ и величина $\omega(L(f), t)$, входящая в запись $\delta_{f}$ и $\sigma_{f}$, в (1.11.2) зависит от $t=D$, а в (1.6.1) - от $t=d$.

\section{§ 2. Устойчивость элемента наилучшего приближения}

Здесь речь пойдет об устойчивости (о непрерывной зависимости от веса $p$ ) элемента наилучшего приближения $l(p, L, f ; x)$ произвольной функции $f \in C$ ( $\Delta=$ $\langle a, b\rangle)$ элементами некоторого чебьшевского подпространства $L \subset C(\Delta)$ с весом $p$.

Напомним, что условие $E(p, L, f)>0$ является достаточным для наличия элемента $l(p, L, f)$ и его единственности для этой функции $f$, условие $W(p, L, f)<\infty-$ для наличия хотя бы одного элемента $l(p, L, f)$ у каждой функции $f \in M(\Delta)$ (но не его единственности) - см. [1], [3].

Для дальнейшего нам понадобится теорема об устойчивости свободы и жесткости суперсистем двух специальных типов.

1.12. При $E \subset \mathbb{R}, t>0$ и $n=1,2,3, \ldots$ обозначим через $X_{n}(E, t)$ множество всех таких наборов $A=\left\{a_{1}, a_{2}, \ldots, a_{n+1}\right\} \subset E$ из $n+1$ точек, что $a_{k+1}-a_{k} \geqslant t$, $k=1, \ldots, n$. Через $\mathrm{p}_{1, n}(E, t)$ обозначим множество всех весов $p$ на $E$, для каждого из которых в $X_{n}(E, t)$ имеется такой набор $A=\left\{a_{k}=a_{k}(p)\right\}$, что $p(x)=(0,0)$ при $x \notin A$, а в точках $a_{k}$ с ростом $k$ вес $p(x)$ принимает попеременно значения $(0,1)$ и $(1,0)$, начиная с любого из них. Через $\mathrm{p}_{2, n}(E, t)$ обозначим множество всех таких весов $p$ на $E$, что

$$
p(x)=\left(p_{-}(x) \equiv \max \left\{q_{-}(x), r_{-}(x)\right\}, p_{+}(x) \equiv \max \left\{q_{+}(x), r_{+}(x)\right\}\right),
$$


где $q=\left(q_{-}, q_{+}\right)$и $r=\left(r_{-}, r_{+}\right)$принадлежат $\mathrm{p}_{1, n}(E, t)$, причем $q\left(a_{1}(q)\right)=(1,0), \mathrm{a}$ $r\left(a_{1}(r)\right)=(0,1)$. Таким образом,

$$
\begin{array}{cl}
p(x)=(0,0) \quad \forall x \notin A(q) \cup A(r), & p_{+}\left(a_{k}(q)\right)=p_{-}\left(a_{k+1}(q)\right)=1, \\
p_{-}\left(a_{k}(r)\right)=p_{+}\left(a_{k+1}(r)\right)=1, & k=1,3,5, \ldots
\end{array}
$$

Очевидно, классы р $1, n(E, t)$ и $\mathrm{p}_{2, n}(E, t)$ пусты одновременно с классом $X_{n}(E, t)$. Если $p \in \mathrm{p}_{2, n}(E, t)$, то число точек $x \in E$, в которых $p_{+}(x)>0$ (т.е. $p_{+}(x)=1$ ), не больше $n+1$ и не меньше $(n+1) / 2$. Ясно также, что если класс $X_{n}(E, t)$ не пуст при $t=T$, то он не пуст и при $0<t \leqslant T$, и что если $E$ состоит не менее чем из $n+1$ точек, то он не пуст при всех достаточно малых $t>0$. Если $L-$ $n$-мерное чебышевское подпространство из $C(\Delta=[a, b])$, то при $0<t<|\Delta| / n$ класс $X_{n}(\Delta, t)$ не пуст и $W(p, L)<\infty$ при $p \in \mathrm{p}_{j, n}(\Delta, t), j=1,2$ (см. п. 0.1).

Для непустого класса $X_{n}(E, t)$ и семейства $L \subset M(E)$ положим

$$
W_{j, n}(L, t):=W\left(\mathrm{p}_{j, n}(E, t), L\right), \quad S_{j, n}(L, t):=S\left(\mathrm{p}_{j, n}(E, t), L\right)
$$

$(j=1,2)$; если $\operatorname{dim} L-$ линейная размерность $L$ (т.е. размерность линейной оболочки $L)$ - есть $n$, то индекс $n$ здесь можно опускать.

Очевидно, $W_{1, n}(L, t)$ и $W_{2, n}(L, t)$ не возрастают (как функции $t$ ), а $S_{1, n}(L, t)$ и $S_{2, n}(L, t)$ не убывают на их области определения. Если класс $X_{n}(E, t)$ не пуст при некотором $t>0$ и пуст при $t=T$ (что имеет место при ограниченности $E$ ), положим

$$
S_{j, n}(L, T):=\sup \left\{S_{j, n}(L, t)\right\}, \quad W_{j, n}(L, T):=\inf \left\{W_{j, n}(L, t)\right\},
$$

где $\sup$ и inf берутся по всем $t>0$, для которых класс $X_{n}(E, t)$ не пуст $(j=1,2)$.

Если $L \subset M(\langle a, b\rangle), \operatorname{dim} L<\infty$, через $\omega^{-1}(t)$ обозначаем функцию, обратную к $t=\omega(L, \delta): \omega^{-1}(t):=\min \{\delta: \omega(L, \delta)=t\}$.

1.13. Лемма. Пусть $\Delta=[a, b], L \subset M(\Delta), n$-натуральное иисло. Тогда при $0<u \leqslant v<|\Delta| / n$ имеем неравенство

$$
0 \leqslant S_{j, n}(L, v)-S_{j, n}(L, u) \leqslant \omega(L, n(v-u)+0)+n(v-u), \quad j=1,2 .
$$

ДокАЗАТЕЛьство. Сначала докажем утверждение

1.13а. При $0<u<v<|\Delta| / n$ для любого $A=\left\{a_{1}, a_{2}, \ldots, a_{n+1}\right\} \in$ $X_{n}(\Delta, u)$ найдется $B=\left\{b_{1}, b_{2}, \ldots, b_{n+1}\right\} \in X_{n}(\Delta, v)$, для которого $\left|b_{k}-a_{k}\right| \leqslant$ $n(v-u) \forall k$.

Для этого положим $h=v-u, b_{1}=\max \left\{a, a_{1}-n h\right\}, b_{k}=\max \left\{b_{k-1}+v, a_{k}-n h\right\}$ при $k=2,3, \ldots, n+1$. Очевидно, $b_{k}-b_{k-1} \geqslant v$ при $1 \leqslant k \leqslant n+1$. Покажем, что 
$b_{n+1} \leqslant b$, что вместе с предыдушим даст $\left\{b_{1}, b_{2}, \ldots, b_{n+1}\right\} \in X_{n}(\Delta, v)$. Очевидно,

$$
\begin{aligned}
& b_{1}+v=\max \left\{a+v, a_{1}+u-(n-1) h\right\} \leqslant \max \left\{a+v, a_{2}-(n-1) h\right\}, \\
& b_{2}=\max \left\{b_{1}+v, a_{2}-n h\right\} \leqslant \max \left\{a+v, a_{2}-(n-1) h, a_{2}-n h\right\} \\
& =\max \left\{a+v, a_{2}-(n-1) h\right\}, \\
& b_{2}+v \leqslant \max \left\{a+2 v, a_{2}+u-(n-2) h\right\} \leqslant \max \left\{a+2 v, a_{3}-(n-2) h\right\} \text {, } \\
& b_{3}=\max \left\{b_{2}+v, a_{3}-n h\right\} \leqslant \max \left\{a+2 v, a_{3}-(n-2) h\right\}, \\
& b_{n+1} \leqslant \max \left\{a+n v, a_{n+1}\right\} \leqslant b .
\end{aligned}
$$

Нетрудно видеть, что $b_{k}-a_{k} \leqslant(k-1) h, k=1,2, \ldots, n+1$. Действительно, это очевидно при $k=1$. Если это верно при некотором $k \geqslant 1$, то

$$
\begin{aligned}
b_{k+1}-a_{k+1} & =\max \left\{\left(b_{k}-a_{k}\right)+v-\left(a_{k+1}-a_{k}\right),-n h\right\} \\
& \leqslant \max \{(k-1) h+h,-n h\}=k h,
\end{aligned}
$$

что и требовалось доказать.

Так как $a_{k}-b_{k} \leqslant n h$, то $\left|b_{k}-a_{k}\right| \leqslant n h$, и 1.13 а доказано.

1.13b. Пусть $\varepsilon>0$, а $p \in \mathrm{p}_{j, n}(\Delta, u)$ таков, что $S(p, L)<S_{j, n}(L, u)+\varepsilon$. По 1.13 a найдется такой вес $q \in \mathrm{p}_{j, n}(\Delta, v)$, что $d(p, q) \leqslant n h$. По 0.4 имеем

$$
S_{j, n}(L, v)-\left(S_{j, n}(L, u)+\varepsilon\right)<S(q, L)-S(p, L) \leqslant \omega(L, n h+0)+n h
$$

откуда и следует (1.13.1).

Напомним определение четности нуля непрерывной функции.

Если в некоторой окрестности $U$ точки $t \in \mathbb{R}$ функция $f(x)$ задана и непрерывна, $f(t)=0, f(t) \neq 0 \quad \forall x \in U \backslash\{t\}$, то $f(x)$ при переходе $x$ через $t$ либо меняет знак, тогда $t$ называем нечетным нулем функиии $f$ и полагаем $n(f, t)=1$, либо не меняет, тогда $t$ называем четным нулем $f$ и полагаем $n(f, t)=2$. Число $n(f, t)$ называют четностью нуля функции $f$ в точке $t$. Если $t$ - концевая точка промежутка $\Delta$, на котором $f(x)$ задана и непрерьвна, $f(t)=0$, а $f(x) \neq 0$ вблизи $t$ при $x \neq t$, то считаем $t$ нечетным нулем и полагаем $n(f, t)=1$.

1.14. ТЕОРемА. Пусть $L$ является либо п-мерным чебышевским подпространством из $C(\Delta=[a, b])($ при $j=1)$, либо (при $j=2)$ таким замкнутым конечномерным конусом в $C(\Delta)$, что у кажсой функции $l \in L$ сумма четностей ее нулей на $\Delta$ не превосходит $n(n \geqslant 1)$. Тогда функции $W_{j, n}(L, t) u$ $S_{j, n}(L, t)(j=1,2)$ непрерывны при $t>0 u$

$$
0 \leqslant S_{j, n}(L, t+h)-S_{j, n}(L, t) \leqslant \omega(L, n h)+n h \quad \forall t>0, \quad \forall h>0 .
$$


ДокАЗАТЕЛЬСтво. Покажем, что $W_{j, k}(L, t)<\infty, j=1,2, t>0$.

1. Пусть сначала $j=1$. По теореме $0.1 W(p, L)<\infty$ для любого $p \in$ $\mathrm{p}_{1, n}(\Delta, t)$. Если бы было $W_{1, n}(L, t)=\infty$, то нашлась бы последовательность весов $p_{k} \in \mathrm{p}_{1, n}(\Delta, t)$, для которой $W\left(p_{k}, L\right) \rightarrow \infty$. В случае необходимости переходя к подпоследовательности, мы можем считать, что $p_{k}$ сходится к некоторому $p \in \mathrm{p}_{1, n}(\Delta, t)$ по $d$-метрике. Но тогда ввиду устойчивости $W(p, L)$ (теорема 0.4 ) было бы $W(p, L)=\infty$.

2. Пусть теперь $j=2$. Покажем, что $W(p, L)<\infty$ для любого $p \in \mathrm{p}_{2, n}(\Delta, t)$. Если бы для такого $p$ было $W(p, L)=\infty$, то (так как $L \cap\{f: f \in C(\Delta),\|f\|=1\}$ компакт) нашелся бы элемент $l \in L$, для которого $\|l\|=1$, и $|\lambda l|_{p, \Delta}=0$ при любом $\lambda=$ const $>0$. Последнее означает (см. (1.12.1)), что все $n+1$ точек $a_{1}, b_{2}, a_{3}, b_{4}, \ldots$, в которых $p_{+}(x)=1$, лежат на $E^{-}:=\{x \in \Delta: l(x) \leqslant 0\}$, а все $n+1$ точек $b_{1}, a_{2}, b_{3}, a_{4}, \ldots$, в которых $p_{-}(x)=1,-$ на $E^{+}:=\{x \in \Delta: l(x) \geqslant 0\}$. Очевидно, $E^{-} \cup E^{+}=\Delta$, а $E^{-} \cap E^{+}-$множество всех нулей функции $l(x)$.

Положим $a_{k}:=a_{k}(q), b_{k}:=a_{k}(r)-$ cм. определение $\mathrm{p}_{2, n}$ в п. 1.12.

a) Будем сначала считать все нули функции $l$ нечетными. Тогда каждое из множеств $E^{-}$и $E^{+}$распадается на конечное число непересекающихся замкнутых отрезков, соответственно будем их называть (-)-отрезками и (+)-отрезками, крайние из которых могут вырождаться в концевые точки отрезка $\Delta$. Очевидно, что (-)-отрезки и $(+)$-отрезки перемежаются на $\Delta$ (одноточечные отрезки, если они имеются, являются крайними) и их обшее число $m$ не превосходит $n+1$. Очевидно также, что на каждом (-)-отрезке лежит не более одной точки множества $A^{+}=\left\{a_{1}, a_{3}, a_{5}, \ldots\right\}$ (на котором $p_{+}(x)=1$ ), а на каждом $(+)$-отрезке - не более одной точки множества $A^{-}=\left\{a_{2}, a_{4}, a_{6}, \ldots\right\}$ (на котором $p_{-}(x)=1$ ). Так как множество $A:=A^{-} \cup A^{+}=\left\{a_{1}, a_{2}, \ldots, a_{n+1}\right\}$ состоит из $n+1$ точек, и $A^{-} \subset E^{+}$, $A^{+} \subset E^{-}$, то $m=n+1$. Занумеруем слева направо подряд все $(-)$-отрезки и $(+)$-отрезки вместе в конечную последовательность $\Delta_{1}, \Delta_{2}, \Delta_{3}, \ldots, \Delta_{n+1}$. Тогда между отрезками $\Delta_{k}$ и точками $a_{k} \in A$ устанавливается взаимно однозначное соответствие, при котором $a_{k} \in \Delta_{k}(k=1,2, \ldots, n+1)$. Подобное соответствие " $b_{k} \in \Delta_{k}$ " устанавливается также между отрезками $\Delta_{k}$ и точками $b_{k} \in B:=$ $\left\{b_{1}, b_{2}, \ldots, b_{n+1}\right\}$. При этом если $\Delta_{k}-(-)$-отрезок, то $p_{+}\left(a_{k}\right)=p_{+}\left(b_{k}\right)=1, \mathrm{a}$ если $\Delta_{k}-(+)$-отрезок, то $p_{-}\left(a_{k}\right)=p_{-}\left(b_{k}\right)=1$. С другой стороны (см. (1.12.1)), имеем равенства: $p_{+}\left(a_{k}\right)=p_{-}\left(b_{k}\right)=1$ при $k=1,3,5, \ldots ; p_{-}\left(a_{k}\right)=p_{+}\left(b_{k}\right)=1$ при $k=2,4,6, \ldots$ Поэтому либо $p_{-}\left(a_{k}\right)=p_{+}\left(a_{k}\right)=1$, либо $p_{-}\left(b_{k}\right)=p_{+}\left(b_{k}\right)=1$ $(k=1,2, \ldots, n+1)$. Отсюда и из определения веса $p$ следует, что $a_{k}=b_{k}, l\left(a_{k}\right)=0$ при всех $k$, так что $l(x)$ имеет не менее $n+1$ нулей. Это противоречит условию теоремы.

b) Пусть теперь функция $l$ имеет хотя бы один четный нуль. Тогда такие нули разбивают содержашие их отрезки, составляюшие $E^{-}$и $E^{+}$, на меньшие невырожденные отрезки, внутри которых $l(x)$ уже сохраняет знак. Составим семейство $D^{-}(-)$-отрезков, включив в него:

1) все отрезки, составляющие $E^{-}$и не содержащие внутри себя нулей функции $l$;

2) все отрезки, на которые были разбиты указанным вьше способом отрезки, составляюшие $E^{-}$(и до разбиения содержавшие четные нули функции $l$ ); 
3) вырожденные в точки отрезки, являюшиеся одновременно четными нулями функции $l$ и внутренними точками $E^{+}$.

Отрезки, составляющие $D^{-}$, назовем (-)-отрезками; определение семейства $D^{+}$и $(+)$-отрезков мы получим, меняя здесь местами знаки - и + . Положив $D=D^{-} \cup D^{+}$, занумеруем отрезки семейства $D$ в последовательность $\left\{\Delta_{k}\right\}$ $(k=1,2, \ldots, m\}$ в порядке их естественного следования слева направо. При этом одноточечный отрезок $\Delta_{k}$ совпадает с правым концом $\Delta_{k-1}$ и левым конщом $\Delta_{k+1}$. Как и выше, между отрезками $\Delta_{k}$ и точками множеств $A$ и $B$ устанавливается взаимно однозначное соответствие по включению $a_{k} \in \Delta_{k}, b_{k} \in \Delta_{k}$, что и в этом случае приводит к противоречию с условием теоремы. Этим доказано, что $W(p, L)<\infty$ для любого $p \in \mathrm{p}_{2, n}(\Delta, t)$.

c) Из неравенства $W(p, L)<\infty \forall p \in \mathrm{p}_{2, n}(\Delta, t)$, как и в п. 1 из неравенства $W(p, L)<\infty \forall p \in \mathrm{p}_{1, n}(\Delta, t)$ мы получили неравенство $W_{1, n}(L, t)<\infty$, получаем неравенство $W_{2, n}(L, t)<\infty(t>0)$.

3. По лемме 1.13 выполняется неравенство (1.14.1), функции $S_{j, n}(L, t)$ непрерывны при $t>0$, а так как $1 / S_{j, n}(L, t)=W_{j, n}(L, t)<\infty$, то при $t>0$ непрерывна и функция $W_{j, n}(L, t)$. Теорема доказана.

1.15. ТеОРемА. Если веса $p, q$ ограничены на $\Delta=[a, b], L-n$-мерное чебьшевское подпространство в $C(\Delta), f \in C(\Delta), E(p, L, f)>0, E(q, L, f)>0$, mo

$$
\begin{aligned}
& \|l(p, L, f)-l(q, L, f)\| \leqslant A \sigma(p, q, L(f) ; d), \quad d=d(p, q), \\
& A=\bar{S}^{-1} W_{1, n}\left(L, \omega^{-1}(2 \bar{S})\right)(E(p, L, f) W(p, L(f))+E(q, L, f) W(q, L(f))) \\
& \quad \leqslant 3 \bar{S}^{-1} W_{1, n}\left(L, \omega^{-1}(2 \bar{S})\right)(W(p, L)+W(q, L)) E(L, f)
\end{aligned}
$$

әде $\bar{S}:=\max \{S(p /\|p\|, L(f)), S(q /\|q\|, L(f))\}, a \quad t=\omega_{f}^{-1}(u)-$ функция, обратная $к u=\omega(L(f), t)\left(\omega_{f}^{-1}(u):=\min \{t: \omega(L(f), t)=u\}\right)$.

ДокаЗАТЕльство. 1. Пусть дополнительно $p, q \in \operatorname{DS}(\Delta)$. Положим $l:=$ $l(p, L, f)$, пусть $x_{1}<x_{2}<\ldots<x_{n+1}$ - точки $p$-альтернанса пары $(l, f)($ см. 0.2$)$, $h=E(p, L, f)$ и для определенности пусть

$$
\begin{aligned}
\left(l\left(x_{2 i-1}\right)-f\left(x_{2 i-1}\right)\right) p_{+}\left(x_{2 i-1}\right) & =h \\
\left(l\left(x_{2 i}\right)-f\left(x_{2 i}\right)\right) p_{-}\left(x_{2 i}\right) & =-h, \quad i=1,2, \ldots
\end{aligned}
$$

Положим еше $m:=l(q, L, f), h^{\prime}=E(q, L, f), P(\cdot)=|\cdot|_{p, \Delta}, Q(\cdot)=|\cdot|_{q, \Delta}$.

2. Из (1.1.2) получаем неравенства

$$
\begin{gathered}
P(m-f) \leqslant Q(m-f)+Q(m-f) y=h^{\prime}+h^{\prime} y \\
h^{\prime} \leqslant Q(l-f) \leqslant P(l-f)+P(l-f) z=h+h z \\
(y=W(q, L(f)) \cdot(\|p\| \omega(L(f), d)+d), z=W(p, L(f)) \cdot(\|q\| \omega(L(f), d)+d)), \\
P(m-f) \leqslant h+\alpha \\
\alpha:=h z+h^{\prime} y \leqslant\left\{h W(p, L(f))+h^{\prime} W(q, L(f))\right\} \sigma(p, q, L(f) ; d) .
\end{gathered}
$$


3. Поэтому

$$
\begin{array}{r}
\left(m\left(x_{2 i-1}\right)-f\left(x_{2 i-1}\right)\right) p_{+}\left(x_{2 i-1}\right) \leqslant h+\alpha, \\
-\left(m\left(x_{2 i}\right)-f\left(x_{2 i}\right)\right) p_{-}\left(x_{2 i}\right) \leqslant h+\alpha .
\end{array}
$$

Почленно вычитая из неравенства (1.15.7) равенство (1.15.3) и прибавляя к неравенству (1.15.8) равенство (1.15.4), получаем

$$
\begin{aligned}
\left(m\left(x_{2 i-1}\right)-l\left(x_{2 i-1}\right)\right) p_{+}\left(x_{2 i-1}\right) & \leqslant \alpha, \\
\left(m\left(x_{2 i}\right)-l\left(x_{2 i}\right)\right) p_{-}\left(x_{2 i}\right) & \geqslant-\alpha .
\end{aligned}
$$

Рассмотрим такой вес $u(x)=\left(u_{-}(x), u_{+}(x)\right)$, что $u_{-}\left(x_{2 i}\right)=p_{-}\left(x_{2 i}\right), u_{-}(x)=0$ при $x \neq x_{2 i}, u_{+}\left(x_{2 i-1}\right)=p_{+}\left(x_{2 i-1}\right), u_{+}(x)=0$ при $x \neq x_{2 i-1} \quad(i=1,2, \ldots)$. По теореме $0.1 W(u, L)<\infty$. Очевидно, $|m-l|_{u, \Delta} \leqslant \alpha$, так что (по определению $W(u, L))$

$$
\|l-m\| \leqslant W(u, L) \cdot \alpha .
$$

Оценим $W(u, L)$. Из (1.15.3) и (1.15.4) имеем

$$
p_{+}\left(x_{2 i-1}\right) \geqslant \frac{h}{\|l-f\|}, \quad p_{-}\left(x_{2 i}\right) \geqslant \frac{h}{\|l-f\|} .
$$

Отсюда и из неравенства $\|l-f\| \leqslant W(p, L(f)) h$ получаем

$$
r:=\min \left\{p_{+}\left(x_{2 i-1}\right), p_{-}\left(x_{2 i}\right): i=1,2, \ldots\right\} \geqslant \frac{1}{W(p, L(f))} .
$$

Положим $v(x)=\left(v_{-}(x), v_{+}(x)\right):=\left(\operatorname{sign} u_{-}(x), \operatorname{sign} u_{+}(x)\right)$ (считаем $\left.\operatorname{sign} 0=0\right)$. Так как $v_{-}(x) \leqslant u_{-}(x) / r, v_{+}(x) \leqslant u_{+}(x) / r$, то (см. $\left.(1.15 .11)\right)$

$$
\begin{aligned}
W(u, L) & =\frac{1}{r} W\left(\frac{u}{r}, L\right) \leqslant W(p, L(f)) \cdot W(v, L) \\
& \leqslant W(p, L(f)) \cdot W_{1, n}(L, \rho), \\
\rho & :=\min \left\{x_{k+1}-x_{k}: k=1, \ldots, n\right\} .
\end{aligned}
$$

4. Заметим теперь, что если $g:=l-f$, то по (1.15.3) и (1.15.4) имеем неравенство

$$
\left|g\left(x_{k+1}\right)-g\left(x_{k}\right)\right| \geqslant \frac{2 h}{\|p\|}, \quad k=1, \ldots, n .
$$

Поэтому $\|g\| \cdot \omega(g /\|g\|, \rho)=\omega(g, \rho) \geqslant 2 h /\|p\|$. Отсюда и из соотношения $\|g\| \leqslant$ $W(p, L(f)) h=h / S(p, L(f))$ получаем неравенства

$$
\begin{gathered}
\omega(L(f), \rho) \geqslant \frac{2 h}{\|p\| \cdot\|g\|} \geqslant 2 S\left(\frac{p}{\|p\|}, L(f)\right), \\
\rho \geqslant \omega_{f}^{-1}\left(2 S\left(\frac{p}{\|p\|}, L(f)\right)\right) .
\end{gathered}
$$


5. Учитывая невозрастание функции $W_{1, n}(L, t)=1 / S_{1, n}(L, t)$, из (1.15.10), $(1.15 .12)$ и (1.15.13) получаем

$$
\|l-m\| \leqslant\left(\frac{1}{S}\left(\frac{p}{\|p\|}, L(f)\right)\right)^{-1} \cdot W_{1, n}\left(L, \omega_{f}^{-1}\left(2 S\left(\frac{p}{\|p\|}, L(f)\right)\right)\right) \alpha .
$$

Если здесь $p$ и $q$ (а также $l$ и $m$ ) поменять местами, то $\|l-m\|$ и $\alpha$ не изменятся, а в остальной части неравенства $p$ заменяется на $q$. Из этих двух неравенств и следует (1.15.1).

6. Выше доказательство начиналось с предположения $p, q \in \mathrm{DS}(\Delta)$, так что если $p, q$ лишь ограничены, то неравенство (1.15.1) верно при замене $p, q$ их регуляризациями $\bar{p}, \bar{q}$ (см. введение). Но так как $d(p, q)=d(\bar{p}, \bar{q})$ (см. [4, теорема 3$])$, а также, очевидно,

$$
\begin{gathered}
\|\bar{r}\|=\|r\|, \quad l(r, L, f)=l(\bar{r}, L, f), \quad E(r, L, f)=E(\bar{r}, L, f), \\
S\left(\frac{\bar{r}}{\|\bar{r}\|}, L(f)\right)=S\left(\frac{r}{\|r\|}, L(f)\right), \quad W\left(\frac{\bar{r}}{\|\bar{r}\|}, L(f)\right)=W\left(\frac{r}{\|r\|}, L(f)\right)
\end{gathered}
$$

при $r=p, q$, то теорема 1.15 доказана полностью.

Напомним, что $\tilde{f}:=f /\|f\|, \tilde{p}:=p /\|p\|, D(p, q):=d(\tilde{p}, \tilde{q})$.

1.16. Теорема. Если веса $p, q$ ограничены на $\Delta=[a, b], L$-n-мерное чебышевское подпространство в $C(\Delta), f \in C(\Delta), E(p, L, f)>0, E(q, L, f)>0$, $d=d(p, q), \quad D=D(\tilde{p}, \tilde{q}), m o$

$$
\begin{aligned}
\| l(p, L, f) & -l(q, L, f) \| \\
\leqslant & M B(W(\tilde{p}, L)+W(\tilde{q}, L)) E(L, f) \Omega(d) \\
& +\frac{3}{2} M B(W(p, L)+W(q, L)) E(L, f) d, \\
\| l(p, L, f)- & l(q, L, f) \| \\
\leqslant & B(W(\tilde{p}, L)+W(\tilde{q}, L)) E(L, f)\left(\Omega(D)+\frac{3}{2} D\right),
\end{aligned}
$$

$2 \partial e$

$$
\begin{aligned}
& M=\max \left\{\frac{\|p\|}{\|q\|}, \frac{\|q\|}{\|p\|}\right\}, \quad \Omega(t)=\omega(L, t)+\omega(\tilde{f}, t), \\
& B=\frac{6}{s} W_{1, n}\left(L, \Omega^{-1}(s)\right), \quad s=\max \{E(\tilde{p}, L, \tilde{f}) S(\tilde{p}, L), E(\tilde{q}, L, \tilde{f}) S(\tilde{q}, L)\},
\end{aligned}
$$

функиия $\Omega^{-1}$ обратна $к \Omega$.

1.16a. ЗАмЕчАнИЕ. Как видно из приводимого ниже доказательства, в этой теореме условие $E(q, L, f)>0$ можно заменить условием $W(q, L)<\infty$ (котороепо лемме 3.3 из [1] слабее условия $E(q, L, f)>0)$. При этом в выражении для $B$ под $s$ нужно будет понимать величину $E(\tilde{p}, L, \tilde{f}) S(\tilde{p}, L)$, а под $l(q, L, f)$ - какой-либо элемент наилучшего приближения из $L$ для $f$ с весом $q$.

Отметим также, что функции $\Omega$ и $\Omega^{-1}$ не зависят от $p$ и $q$.

ДоКАЗАТЕЛЬСТВО ТЕОРЕМЫ 1.16 аналогично доказательству теоремы 1.15. Укажем по пунктам те изменения, которые при этом нужно произвести. 
1. Оставить без изменений.

2. По (1.1.0) имеем

$$
\begin{aligned}
& P(m-f) \leqslant Q(m-f)+\|p\| \omega(m-f, d)+\|m-f\| d, \\
& Q(m-f) \leqslant Q(l-f) \leqslant P(l-f)+\|q\| \omega(l-f, d)+\|l-f\| d,
\end{aligned}
$$

откуда

$$
\begin{aligned}
P(m-f) \leqslant & h+\|q\| \omega(l-f, d)+\|l-f\| d+\|p\| \omega(m-f, d)+\|m-f\| d \\
\leqslant & +\|q\| \omega(l, d)+\|p\| \omega(m, d)+(\|q\|+\|p\|) \omega(f, d) \\
& +(\|l-f\|+\|m-f\|) d \\
\leqslant & h+(\|q\| \cdot\|l\|+\|p\| \cdot\|m\|) \omega(L, d) \\
& +(\|q\|+\|p\|) \omega(f, d)+(\|l-f\|+\|m-f\|) d
\end{aligned}
$$

Так как

$$
\begin{gathered}
\|l\| \leqslant W(p, L) P(l) \leqslant W(p, L)(P(l-f)+P(f)) \leqslant 2\|p\| W(p, L)\|f\| \\
(P(l-f)=h \leqslant P(0-f) \leqslant\|f\| \cdot\|p\|), \text { то } \\
\|l\| \leqslant 2\|p\| W(p, L)\|f\|=2 W(\tilde{p}, L)\|f\|
\end{gathered}
$$

и, аналогично,

$$
\|m\| \leqslant 2\|q\| W(q, L)\|f\|=2 W(\tilde{q}, L)\|f\| .
$$

Так как $W(\varkappa, L) \geqslant 1$ при $\|\varkappa\|=1$, то отсюда

$$
\|l-f\| \leqslant 3 W(\tilde{p}, L)\|f\|, \quad\|m-f\| \leqslant 3 W(\tilde{q}, L)\|f\| .
$$

Из (1.16.2)-(1.16.5) получаем неравенство

$$
\begin{aligned}
P(m-f) \leqslant & h+2\|f\|(\|q\| W(\tilde{p}, L)+\|p\| W(\tilde{q}, L)) \omega(L, d) \\
& +(\|q\|+\|p\|) \omega(f, d)+3\|f\|(W(\tilde{p}, L)+W(\tilde{q}, L)) d \\
\leqslant & h+\alpha, \\
\alpha= & 2\|f\|(\|q\| W(\tilde{p}, L)+\|p\| W(\tilde{q}, L))(\omega(L, d)+\omega(\tilde{f}, d)) \\
& +3\|f\|(\|p\| W(p, L)+\|q\| W(q, L)) d
\end{aligned}
$$

(мы воспользовались тем, что $W(\varkappa, L) \geqslant 1$ при $\|\varkappa\|=1$, откуда $\|p\|+\|q\| \leqslant$ $\|p\| W(\tilde{q}, L)+\|q\| W(\tilde{p}, L))$. Положив $M=\max \{\|p\| /\|q\|,\|q\| /\|p\|\}$, отсюда получаем

$$
\begin{aligned}
\max \left\{\frac{\alpha}{\|f\| \cdot\|p\|}, \frac{\alpha}{\|f\| \cdot\|q\|}\right\} \leqslant & 2 M(W(\tilde{p}, L)+W(\tilde{q}, L))(\omega(L, d)+\omega(\tilde{f}, d)) \\
& +3 M(W(p, L)+W(q, L)) d
\end{aligned}
$$


3. Не изменяется.

4. Заметим, что для $g:=l-f$ из (1.15.3) и (1.15.4) получаем неравенства

$$
\left|g\left(x_{k+1}\right)-g\left(x_{k}\right)\right| \geqslant \frac{2 h}{\|p\|}, \quad k=1, \ldots, n, \quad \omega(g, \rho) \geqslant \frac{2 h}{\|p\|} .
$$

Отсюда, учитывая (1.16.3) и неравенство $W(\tilde{p}, L) \geqslant 1$, имеем оценки

$$
\begin{aligned}
2 E(\tilde{p}, L, f) & =\frac{2 h}{\|p\|} \leqslant \omega(l-f, \rho) \leqslant \omega(l, \rho)+\omega(f, \rho) \\
& \leqslant\|l\| \omega(L, \rho)+\|f\| \omega\left(\frac{f}{\|f\|}, \rho\right) \leqslant 2\|f\| W(\tilde{p}, L) \Omega(\rho), \\
\Omega(\rho) & :=\omega(L, \rho)+\omega(\tilde{f}, \rho) \geqslant E(\tilde{p}, L, \tilde{f}) S(\tilde{p}, L), \\
\rho & \geqslant \Omega^{-1}(E(\tilde{p}, L, \tilde{f}) S(\tilde{p}, L)) .
\end{aligned}
$$

5. Из (1.15.10), (1.15.12), (1.16.7) и (0.3.1) получаем

$$
\begin{aligned}
\|l-m\| & \leqslant W(p, L(f)) W_{1, n}\left(L, \Omega^{-1}(E(\tilde{p}, L, \tilde{f}) S(\tilde{p}, L))\right) \alpha \\
& \leqslant 3 s_{p}^{-1} \cdot W_{1, n}\left(L, \Omega^{-1}\left(s_{p}\right)\right) E(L, f) \frac{\alpha}{\|f\| \cdot\|p\|}, \\
s_{p} & :=E(\tilde{p}, L, \tilde{f}) S(\tilde{p}, L) .
\end{aligned}
$$

Меняя здесь местами $p$ и $q, l$ и $m$, получим еще одно неравенство. Учитывая убьвание функции $(1 / s) W_{1, n}\left(L, \Omega^{-1}(s)\right)$, из этих неравенств и из (1.16.7) получаем (1.16.1a) и (1.16.1b).

6. Без изменений.

Из замечания 1.16 а и теоремы 0.4 получается

1.17. Теорема. Пусть р и q-нормированнье веса на $\Delta=[a, b], L$ - чебьшевское подпространство на $\Delta, \omega(L, d)+d \leqslant(1-k) S(p, L)$ при некотором $k \in(0,1)$. Тогда если $f \in C(\Delta)$ u $E(p, L, f)>0$, mo

$$
\|l(p, L, f)-l(q, L, f)\| \leqslant \frac{C}{k}\left\{\omega(L, d)+\omega\left(\frac{f}{\|f\|}, d\right)+d\right\}, \quad d=d(p, q),
$$

где $C=C(p, L, f)$ не зависит от $q, d u k$.

Отметим, что из чебьшевости $L$ и условия $E(p, L, f)>0$ следует сушествование и единственность $l(p, L, f)$ (см. $[1$, п. 3.8], $[2$, п. 2]). При этом элемент $l(q, L, f)$ (при условии $\omega(L, d)+d \leqslant(1-k) S(p, L)$ его сушествование следует из конечности $W(q, L)$ - см. (0.4.2)) может быть не единственным, и тогда, конечно, неравенство (1.17.1) вьполняется для каждого элемента $l(q, L, f)$. Единственность же $l(q, L, f)$ при $E(p, L, f)>0$ имеется, например, если $d=d(p, q)$ столь мало, что правая часть неравенства (1.8.1) меньше $E(p, L, f)$, так как тогда $E(q, L, f)>0$. 


\section{§ 3. Случай подпространства, чебышевского на полуинтервале. Главный элемент наилучшего приближения}

Здесь мы приведем обобщения некоторых результатов, полученных вьше для случая чебышевских подпространств $L$ на отрезке $\Delta=[a, b]$, на случай подпространств $L \subset C(\Delta)$, чебышевских лишь на полуинтервале $[a, b)$ или $(a, b]$. Эти обобщения используются в приводимых в гл. 2 приложениях знакочувствительных аппроксимаций к теории ужей.

1.18. ОПРЕДЕЛЕНИЕ. Пусть $L-n$-мерное подпространство в $C(\Delta=[a, b])$, чебышевское на интервале $(a, b), p$ - ограниченный вес на $\Delta, p \neq \equiv(0,0), f \in C(\Delta) \backslash L$. Элемент наилучшего приближения $l(p, L, f)$ называется индуцированным әлементом наилучшего приближения функции $f$ подпространством $L$ с весом $p$, если существует последовательность весов $\left\{q_{k}\right\}_{k=1}^{\infty}$, для которых $\operatorname{supp}\left(q_{k}\right) \subset$ $(a, b) \forall k, d\left(q_{k}, p\right) \rightarrow 0$ и $\left\|l\left(q_{k}, L, f\right)-l(p, L, f)\right\| \rightarrow 0$ при $k \rightarrow \infty\left(\|\cdot\|=\|\cdot\|_{C(\Delta)}\right)$.

1.19. Лемма. Пусть $L-n$-мерное подпространство в $C(\Delta=[a, b])$, чебышевское на интервале $(a, b), \quad p \in \operatorname{DS}(\Delta), f \in C(\Delta) \backslash L, W(p, L(f))<\infty$. Тогда $f$ имеет в $L$ по крайней мере один индуцированный әлемент наилучшего приближсения с весом $р$.

ДоказАТЕЛЬСтво. Не ограничивая общности, считаем $\|p\|=1$. При $0<$ $\delta<(b-a) / 2$ через $\psi(\delta ; x)$ обозначим линейное отображение отрезка $[a+\delta, b-\delta]$ на $[a, b]$ такое, что $\psi(\delta ; a+\delta)=a, \psi(\delta ; b-\delta)=b$. Пусть $0<\delta_{k}<(b-a) / 2$, $\delta_{k} \rightarrow 0(k \rightarrow \infty), q_{k}(x)=p\left(\psi\left(\delta_{k} ; x\right)\right)$ при $x \in \Delta_{k}:=\left[a+\delta_{k}, b-\delta_{k}\right], q_{k}(x)=0$ при $x \in \Delta \backslash \Delta_{k}$. Тогда $\operatorname{supp}\left(q_{k}\right) \subset(a, b)$, при $k \rightarrow \infty$ имеем $d\left(q_{k}, p\right) \rightarrow 0$, $W\left(q_{k}, L(f)\right)<\infty$ (см. п. 0.4). При этих $k$ сушествуют $l\left(q_{k}, L, f\right)=: l_{k}-$ см. введение. Из определения $W\left(q_{k}, L(f)\right)$, из п. 0.4 и п. 1.8 получаем

$$
\left\|l_{k}-f\right\| \leqslant W\left(q_{k}, L(f)\right) E\left(q_{k}, L, f\right) \rightarrow W(p, L(f)) E(p, L, f)=: M<\infty,
$$

так что последовательность $\left\{l_{k}\right\} \subset L$ ограничена. Пусть $l^{*}$ - какой-либо ее предельный элемент. Можно считать, что $\left\|l^{*}-l_{k}\right\| \rightarrow 0$. Положим $g=l^{*}-f$, $g_{k}=l_{k}-f\left(\left\|g_{k}-g\right\| \rightarrow 0\right), P(\cdot)=|\cdot|_{p, \Delta}, q=q_{k}, Q_{k}(\cdot)=|\cdot|_{q, \Delta}\left(\left\|q_{k}\right\|=\left\|Q_{k}\right\|=1\right)$, $d_{k}=d\left(q_{k}, p\right)$. Пользуясь выпуклостью функционала $Q_{k}$ и неравенством (1.1.1) с $Q=Q_{k}, l=g$ и $d=d_{k}$, получаем

$$
\begin{aligned}
\left|Q_{k}\left(g_{k}\right)-P(g)\right| & \leqslant\left|Q_{k}\left(g_{k}\right)-Q_{k}(g)\right|+\left|Q_{k}(g)-P(g)\right| \\
& \leqslant\left\|g_{k}-g\right\|+\omega\left(g, d_{k}\right)+\|g\| d_{k} \rightarrow 0
\end{aligned}
$$

$(k \rightarrow \infty)$. Отсюда $P\left(l^{*}-f\right)=\lim Q_{k}\left(g_{k}\right)=\lim E\left(q_{k}, L, f\right)=E(p, L, f)$ (см. п. 1.8), так что $l^{*}=l(p, L, f)$. Лемма доказана.

1.20. ЛЕмма. При условиях леммы 1.19 каждый индуцированный әлемент наилучшего приближения $l^{*}=l(p, L, f)$ имеет с $f(n+1)$-точечный р-альтернанс на $\Delta$.

4 Серия математическая, №3 
ДоКАЗАТЕЛЬСТВо. В ходе доказательства нам понадобится следующее свойство сходимости по $d$-расстоянию, вытекаюшее из рассуждений доказательства теоремы 5 работы $[4]$.

Если последовательность функций $f_{k}$, определенных и полунепрерывных сверху на некотором множестве $E \subset \mathbb{R}$, сходится по $d$-метрике к функции $f$, определенной и полунепрерывный сверху на $E$, а последовательность точек $x_{k} \in E$ сходится $x$ некоторой точке $t \in E$, mo $\overline{\lim }_{k \rightarrow \infty} f_{k}\left(x_{k}\right) \leqslant f(t)$.

Ниже будем придерживаться обозначений, принятых в доказательстве леммы 1.19 , и предположения $\|p\|=1$.

Пусть $q_{k}=\left(q_{k_{-}}, q_{k_{+}}\right), \quad h=E(p, L, f)$. Заметим, что у каждой пары $l_{k}, f$ имеется $(n+1)$-точечный $q_{k}$-альтернанс $x_{1}(k)<x_{2}(k)<\ldots<x_{n+1}(k)$ на $\Delta_{k} \subset$ $(a, b)$ (см. п. 0.2$)$ и (ввиду неравенств $\left.0<q_{k \pm}\left(x_{j}(k)\right) \leqslant 1\right)$ при всех достаточно больших $k\left(k \geqslant K^{\prime}\right)$ вьполняется соотношение

$$
\left|g_{k}\left(x_{j}(k)\right)\right| \geqslant E\left(q_{k}, L, f\right)>\frac{2}{3} h, \quad j=1, \ldots, n+1 .
$$

Так как $\left\|g_{k}-g\right\|=\left\|l_{k}-l^{*}\right\| \rightarrow 0(k \rightarrow \infty)$, то $\left\|g_{k}-g\right\|<h / 12$ при всех достаточно больших $k\left(k \geqslant K^{\prime \prime}\right)$.

Фиксируем $k \geqslant K=\max \left\{K^{\prime}, K^{\prime \prime}\right\}$. Открытое множество $U$ определим так:

$$
U=\left\{x: x \in(a, b),|g(x)|>\frac{h}{3}\right\}
$$

Для длины $|\gamma|$ каждого из интервалов $\gamma=(\alpha, \beta)$, составляющих $U$ и содержащих хотя бы одну точку $x_{j}(k)$, по (1.20.1) имеем, например, при $\beta \in(a, b), g(\beta)=h / 3$

$$
\omega(|\gamma|):=\omega(g,|\gamma|) \geqslant\left|g\left(x_{j}(k)\right)\right|-|g(\beta)| \geqslant\left(\left|g_{k}\left(x_{j}(k)\right)\right|-\frac{h}{12}\right)-\frac{h}{3}>\frac{h}{4},
$$

$|\gamma|>\omega^{-1}(h / 4)$. Поэтому число таких $\gamma$ не превосходит $|\Delta| / \omega^{-1}(h / 4)$. Еще отметим, что знаки величин $g_{k}\left(x_{j+1}(k)\right)$ и $g_{k}\left(x_{j}(k)\right), j=1,2, \ldots$, противоположны, а их модули превосходят $2 h / 3$, и если $x \in \gamma \ni x_{i}(k), i=1,2, \ldots$, то

$$
\left|g_{k}(x)\right|>|g(x)|-\frac{h}{12}>\frac{h}{4} .
$$

Поэтому интервалы $\gamma$, содержашие соседние точки $x_{i}(k)$, находятся на положительном расстоянии друг от друга. Из сказанного следует, что найдутся такой набор $\left\{\gamma_{1}, \gamma_{2}, \ldots, \gamma_{n+1}\right\}$ из $n+1$ этих интервалов $\gamma$, попарно находящихся на положительном расстоянии друг от друга, и такая подпоследовательность последовательности $\left\{g_{k}\right\}$, которую мы также обозначим через $\left\{g_{k}\right\}_{k=K}^{\infty}$, что $x_{j}(k) \in \gamma_{j}$ для любых $k \geqslant K$ и $j=1, \ldots, n-1$.

Фиксируем $j$ и обозначим через $t_{j}$ какую-либо предельную точку последовательности $\left\{x_{j}(k)\right\}_{k=K}^{\infty}$. Будем считать последовательность $\left\{g_{k}\right\}$ выбранной так, что $x_{k}:=x_{j}(k) \rightarrow t_{j}$ при $k \rightarrow \infty\left(t_{j} \in \bar{\gamma}_{j}\right)$. Пусть для определенности

$$
\left(g_{k}, q_{k}\right)\left(x_{k}\right)=g\left(x_{k}\right) q_{k+}\left(x_{k}\right)=E\left(q_{k}, L, f\right) .
$$


Так как $\left\|g_{k}-g\right\| \rightarrow 0$ при $k \rightarrow \infty$, то из замечания в начале доказательства получаем

$$
h=\lim E\left(q_{k}, L, f\right)=\lim g_{k}\left(x_{k}\right) q_{k+}\left(x_{k}\right) \leqslant g\left(t_{j}\right) \varlimsup q_{k+}\left(x_{k}\right) \leqslant g\left(t_{j}\right) p_{+}\left(t_{j}\right) \leqslant h .
$$

Отсюда $g\left(t_{j}\right) p_{+}\left(t_{j}\right)=h=E(p, L, f)$. Этим доказано наличие у пары $l^{*}, f$ $(n+1)$-точечного $p$-альтернанса на $\Delta$. Лемма доказана.

1.21. Лемма. Если при условиях леммы 1.19 каждая функиия $l \in L$ $(l \not \equiv 0)$ имеет на $\Delta$ не более $n$ геометрически различных нулей, то у $f$ имеется ровно один әлемент наилучшего приближения $l(p, L, f)$, имеющий $c f$ $(n+1)$-точечный р-альтернанс на $\Delta$.

Доказательство этой леммы мы не приводим, поскольку оно основано на стандартном чебьшевском методе подсчета нулей функции $l_{1}(x)-l_{2}(x)$ (т.е. числа точек пересечения графииков функций $l_{1}(x)$ и $\left.l_{2}(x)\right)$, где элементы наилучшего приближения $l_{j}=l_{j}(p, l, f) \quad(j=1,2)$ имеют с $f(n+1)$-точечные $p$-альтернансы. Этот подсчет показывает, что функция $l_{1}-l_{2} \in L$ имеет на $\Delta$ не менее $n+1$ нулей, так что $l_{1}-l_{2} \equiv 0$.

1.22. ОПРЕДЕлЕНИЕ. Пусть $L-n$-мерное линейное подпространство из $C(\Delta=$ $[a, b]), p \in \operatorname{DS}(\Delta), f \in C(\Delta) \backslash L$. Элемент наилучшего приближения $l(p, L, f)$ называется главным әлементом наилучшего приближения функции $f$ элементами подпространства $L$ с весом $p$, если в $A(p, L, f)$ он является единственным, имеющим с $f(n+1)$-точечный $p$-альтернанс на $\Delta$.

Таким образом, для заданной тройки $p, L, f$ может сушествовать не более одного главного элемента наилучшего приближения.

Из лемм 1.19-1.21 получается

1.23. ТЕОРЕМА. Если $L$ - конечномерное линейное подпространство в $C(\Delta=[a, b])$, чебьшевское на $(a, b]$ или на $[a, b), \quad p \in \operatorname{DS}(\Delta), f \in C(\Delta) \backslash L$, $W(p, L(f))<\infty$, то в $A(p, L, f)$ имеется главный әлемент наилучшего приближения $l^{*}(p, L, f)$.

1.24. ОПРЕДЕЛЕНИЕ. Пусть $E \subset \mathbb{R}, f, l, m \in M(E), p$ и $q$ - знакочувствительные веса на $E, x_{1}<x_{2}<\ldots<x_{r}$ и $y_{1}<y_{2}<\ldots<y_{s}$ - альтернансы пар $(l, f)$ и $(m, f)$ соответственно (см. введение). Назовем эти альтернансы синхроннымми $n$-точечными, если $r=s=n$ и разности $l\left(x_{1}\right)-f\left(x_{1}\right)$ и $m\left(y_{1}\right)-f\left(y_{1}\right)$ имеют одинаковые знаки.

1.25. ТеОремА. Если $L$ - $n$-мерное линейное подпространство в $C(\Delta=[a, b])$, чебышевское на $(a, b]$ или $[a, b), \quad f \in C(\Delta) \backslash L, \quad p, q \in \operatorname{DS}(\Delta)$, $W(p, L(f))<\infty, \quad W(q, L(f))<\infty$, главные әлементы наилучшего приближения $l=l(p, L, f)$ и $m=l(q, L, f)$ имеют с $f$ синхронные $(n+1)$-точечные p- и q-альтернансы соответственно, то

$$
\|l-m\| \leqslant A \sigma(p, q, L(f) ; d)=A(N \omega(L(f), d)+d), \quad d=d(p, q),
$$


əде

$$
\begin{gathered}
A=\frac{1}{\underline{s}} W_{2, n}\left(L, \omega^{-1}(2 \underline{s})\right)(E(p, L, f) W(p, L(f))+E(q, L, f) W(q, L(f))), \\
\underline{s}=\min \left\{S\left(\frac{p}{\|p\|}, L(f)\right), S\left(\frac{q}{\|q\|}, L(f)\right)\right\},
\end{gathered}
$$

функиия $t=\omega^{-1}(s)$ обратна к функиии $s=\omega(L(f), t), \quad N=\max \{\|p\|,\|q\|\}$.

ДокаЗАТЕЛЬСТво. Пусть $h=E(p, l, f), h^{\prime}=E(q, l, f), P(\cdot)=|\cdot|_{p, \Delta}$, $Q(\cdot)=|\cdot|_{q, \Delta}$. Так как $f \notin L$ и $W(p, L(f))<\infty$, то $h>0$. Действительно,

$$
0<\|l-f\| \leqslant W(p, L(f))|l-f|_{p, \Delta}=W(p, L(f)) h, \quad l=l(p, L, f) .
$$

Аналогично, $h^{\prime}>0$. По (1.1.2) имеем

$$
\begin{aligned}
& h \leqslant P(m-f) \leqslant Q(m-f)+Q(m-f) y=h^{\prime}+h^{\prime} y, \\
& y= W(q, L(f))(\|p\| \cdot \omega(L(f), d)+d), \\
& h^{\prime} \leqslant Q(l-f) \leqslant P(l-f)+P(l-f) z=h+h z, \\
& z:=W(q, L(f))(\|q\| \cdot \omega(L(f), d)+d), \\
& P(m-f) \leqslant h+\left(h z+h^{\prime} y\right)=h+\alpha, \\
& Q(m-f) \leqslant h^{\prime}+\left(h z+h^{\prime} y\right)=h^{\prime}+\alpha, \\
& \alpha=\left\{\|q\| h W(p, L(f))+\|p\| h^{\prime} W(q, L(f))\right\} \omega(L(f), d) \\
& \quad+\left\{h W(p, L(f))+h^{\prime} W(q, L(f))\right\} d \\
& \leqslant\left\{h W(p, L(f))+h^{\prime} W(q, L(f))\right\} \sigma(p, q, L(f) ; d) .
\end{aligned}
$$

Пусть $x_{1}<x_{2}<\ldots<x_{n+1}$ - точки некоторого $p$-альтернанса пары $(l, f)$, $y_{1}<y_{2}<\ldots<y_{n+1}$ - точки $q$-альтернанса пары $(m, f)$, и, для определенности, пусть

$$
\begin{aligned}
\left(l\left(x_{2 i-1}\right)-f\left(x_{2 i-1}\right)\right) p_{+}\left(x_{2 i-1}\right) & =h, \\
\left(l\left(x_{2 i}\right)-f\left(x_{2 i}\right)\right) p_{-}\left(x_{2 i}\right) & =-h, \\
\left(m\left(y_{2 i-1}\right)-f\left(y_{2 i-1}\right)\right) q_{+}\left(y_{2 i-1}\right) & =h^{\prime}, \\
\left(m\left(y_{2 i}\right)-f\left(y_{2 i}\right)\right) q_{-}\left(y_{2 i}\right) & =-h^{\prime}, \\
i=1,2, \ldots &
\end{aligned}
$$

Из (1.25.2) и (1.25.3) имеем также

$$
\begin{aligned}
\left(m\left(x_{2 i-1}\right)-f\left(x_{2 i-1}\right)\right) p_{+}\left(x_{2 i-1}\right) & \leqslant h+\alpha, \\
-\left(m\left(x_{2 i}\right)-f\left(x_{2 i}\right)\right) p_{-}\left(x_{2 i}\right) & \leqslant h+\alpha, \\
\left(l\left(y_{2 i-1}\right)-f\left(y_{2 i-1}\right)\right) q_{+}\left(y_{2 i-1}\right) & \leqslant h^{\prime}+\alpha, \\
-\left(l\left(y_{2 i}\right)-f\left(y_{2 i}\right)\right) q_{-}\left(y_{2 i}\right) & \leqslant h^{\prime}+\alpha .
\end{aligned}
$$


Почленно вычитая из неравенства (1.25.9) равенство (1.25.5) и прибавляя к неравенству (1.25.10) равенство (1.25.6), получим

$$
\left(m\left(x_{2 i-1}\right)-l\left(x_{2 i-1}\right)\right) p_{+}\left(x_{2 i-1}\right) \leqslant \alpha, \quad\left(l\left(x_{2 i}\right)-m\left(x_{2 i}\right)\right) p_{-}\left(x_{2 i}\right) \leqslant \alpha .
$$

Аналогично, из (1.25.11), (1.25.7) и (1.25.12), (1.25.8) получим

$$
\left(l\left(y_{2 i-1}\right)-m\left(y_{2 i-1}\right)\right) q_{+}\left(y_{2 i-1}\right) \leqslant \alpha, \quad\left(m\left(y_{2 i}\right)-l\left(y_{2 i}\right)\right) q_{-}\left(y_{2 i}\right) \leqslant \alpha .
$$

Обозначим через $v=\left(v_{-}, v_{+}\right)$такой вес, что $v_{+}\left(x_{k}\right)=v_{-}\left(x_{k+1}\right)=1, v_{-}\left(y_{k}\right)=$ $v_{+}\left(y_{k+1}\right)=1, k=1, \ldots, n$, а в остальных случаях $v_{+}(x)=0, v_{-}(x)=0, x \in \Delta$. Положим

$$
r:=\min \left\{p_{+}\left(x_{2 i-1}\right), p_{-}\left(x_{2 i}\right), q_{+}\left(y_{2 i-1}\right), q_{-}\left(y_{2 i}\right): i=1, \ldots, n\right\} .
$$

Тогда из (1.25.13) и (1.25.14) следует, что $|m-l|_{v, \Delta} \leqslant \alpha / r$. Отсюда и из определения $W(v, L)$ получаем неравенство

$$
\|m-l\| \leqslant \frac{\alpha}{r} W(v, L) .
$$

Из (1.25.5)-(1.25.8) имеем $\|l-f\| p_{+}\left(x_{2 i-1}\right) \geqslant h, \ldots$, так что

$$
r \geqslant \min \left\{\frac{h}{\|l-f\|}, \frac{h^{\prime}}{\|m-f\|}\right\} \text {. }
$$

Так как $\|l-f\| \leqslant W(p, L(f)) h,\|m-f\| \leqslant W(q, L(f)) h^{\prime}$, то отсюда

$$
r \geqslant \min \{S(p, L(f)), S(q, L(f))\}=: \underline{s} .
$$

Положим

$$
\begin{gathered}
\lambda:=l-f, \quad \mu:=m-f, \\
X=\min \left\{x_{k+1}-x_{k}: k=1, \ldots, n\right\}, \quad Y=\min \left\{y_{k+1}-y_{k}: k=1, \ldots, n\right\}, \\
\rho=\min \{X, Y\} .
\end{gathered}
$$

Из (1.25.5)-(1.25.8) имеем соответственно

$$
\left|\lambda\left(x_{k+1}\right)-\lambda\left(x_{k}\right)\right| \geqslant \frac{2 h}{\|p\|}, \quad\left|\mu\left(y_{k+1}\right)-\mu\left(y_{k}\right)\right| \geqslant \frac{2 h^{\prime}}{\|q\|} .
$$

Поэтому $\|\lambda\| \omega(\lambda /\|\lambda\|, X) \geqslant 2 h /\|p\|,\|\mu\| \omega(\mu /\|\mu\|, Y) \geqslant 2 h^{\prime} /\|q\|$. Отсюда и из неравенств $\|\lambda\| \leqslant W(p, L(f)) h$ и $\|\mu\| \leqslant W(q, L(f)) h^{\prime}$ получаем

$$
\begin{gathered}
\omega(L(f), X) \geqslant 2 S\left(\frac{p}{\|p\|}, L(f)\right), \quad \omega(L(f), Y) \geqslant 2 S\left(\frac{q}{\|q\|}, L(f)\right), \\
\rho \geqslant \omega^{-1}(2 \underline{s}) .
\end{gathered}
$$

Так как $W(v, L) \leqslant W_{2, n}(L, \rho)$ (см. п. 1.12), функция $W_{2, n}(L, t)$ не возрастает, то из (1.25.15)-(1.25.18) имеем

$$
\|l-m\| \leqslant \frac{\alpha}{\underline{s}} W_{2, n}\left(L, \omega^{-1}(2 \underline{s})\right)
$$

(см. (1.25.4)). Теорема доказана. 


\section{ГлАВА 2. Приложения: некоторые экстремальные задачи в пространствах полиномов}

\section{§ 1. Приложения к теории ужей}

2.0. Полиномиальные ужи были введены С. Карлином [5] как обобшение полиномов Чебьшева $T_{n}(x)=\cos (n \cdot \arccos x)$ (см. также [6]). Подобно полиномам Чебьшева, ужи решают ряд экстремальных задач в классах полиномов. Напомним определение ужа, заодно обобшив его на каналы ("коридоры") с границами разрывными, а также бесконечными. С нашей точки зрения только рассмотрение каналов с полунепрерывными и не обязательно конечными границами придает теории ужей законченный вид.

Ниже $\mathrm{TCH}_{n}(\Delta)$ обозначает семейство всех $n$-мерных чебьшевских подпространств на промежутке $\Delta=\langle a, b\rangle(-\infty \leqslant a<b \leqslant+\infty)$.

2.0а. ОПРЕДЕЛЕНИЕ. Пусть при $n \geqslant 1$ на отрезке $\Delta$ задано $n$-мерное чебышевское подпространство $L\left(L \in \mathrm{TCH}_{n}(\Delta)\right)$ и пара таких функций $g$ и $G$, что $-\infty \leqslant g(x)<G(x) \leqslant+\infty$ при всех $x \in \Delta$ и любых значениях функций $g, G$. Тогда элемент $l^{*} \in L$ назовем ужом из $L$, порожденным парой $(g, G)$, если при всех $x \in \Delta$ выполнено неравенство $g(x) \leqslant l^{*}(x) \leqslant G(x)$ и на $\Delta$ имеется такой набор из $m=n$ точек $x_{1}<x_{2}<x_{3}<\ldots<x_{m}$, что с ростом $k$ от 1 до $m$ точки $M_{k}=\left(x_{k}, l^{*}\left(x_{k}\right)\right)$ попеременно попадают то на дополненный график $F(G)$ функции $G$, то на дополненный график $F(g)$ функции $g$ (начиная с любого из этих множеств). Такой набор $\left\{x_{k}\right\}$ будем называть альтернансом ужа $l^{*}$. Если же имеется альтернанс из $m>n$ точек, то будем называть его избыточным альтернансом ужа $l^{*}$, а сам $l^{*}-$ уникальныцм (или избыточныцм) ужом.

Очевидно, в этом определении функцию $G(x)$ можно заменить ее нижней функцией Бэра $\underline{G}(x)$, а $g(x)$ - ее верхней функцией Бэра $\bar{g}(x)$ (см. введение). Как известно, нижняя функция Бэра полунепрерывна снизу, верхняя - сверху. Отсюда легко увидеть, что при определении ужа из $L \in \mathrm{TCH}_{n}(\Delta)$, порожденного парой $(g, G)$, можно было с самого начала считать $G$ однозначной функцией, полунепрерывной снизу, а $g$ - однозначной функцией, полунепрерьвной сверху (опустив требование дополненности их графиков). Именно так мы и будем считать.

2.0b. ОПРЕДЕЛЕНИЕ. Компактифицируем плоскость $O x y$, присоединив к ней несобственные точки вида $(x,-\infty),(x,+\infty),(-\infty, y)$ и $(+\infty, y)$, где $-\infty \leqslant x$, $y \leqslant+\infty$, и определив сходимость на таком расширении $\left[\mathbb{R}^{2}\right]=[-\infty,+\infty] \times$ $[-\infty,+\infty]$ плоскости $\mathbb{R}^{2}$ покоординатно:

$$
\left(x_{k}, y_{k}\right) \rightarrow(x, y) \Leftrightarrow x_{k} \rightarrow x, \quad y_{k} \rightarrow y, \quad k \rightarrow \infty, \quad-\infty \leqslant x, x_{k}, y, y_{k} \leqslant+\infty .
$$

При указанных выше условиях на $\Delta=\langle a, b\rangle, g, G$ и $L$ обозначим через $B^{0}(g, G)$ односвязную область на $\left[\mathbb{R}^{2}\right]$, ограниченную снизу и сверху дополненными графиками $F(g)$ и $F(G)$ функций $g$ и $G$, а слева и справа - открытыми отрезками на вертикалях $x=a$ и $x=b$ соответственно. Присоединив к $B^{0}(g, G)$ эти открытые отрезки, получим канал $B(g, G)$ с граничными функциями $g$ и $G$. Если $\partial B^{0}(g, G)-$ 
граница $B^{0}(g, G)$ на $\left[\mathbb{R}^{2}\right]$, то $\partial^{-} B(g, G):=F(g) \cap \partial B^{0}(g, G)=F(g)$ назовем нижним берегом канала $B(g, G), \partial^{+} B(g, G):=F(g) \cap \partial B^{0}(g, G)=F(G)$ - его верхним берегом. Отметим, что каждый из берегов - связный компакт (континуум) на $\left[\mathbb{R}^{2}\right]$. Положим еще

$$
L(g, G):=\{l \in L: g(x) \leqslant l(x) \leqslant G(x) \forall x \in \Delta\}
$$

Очевидно, равенство $g(t)=-\infty(t \in \Delta)$ означает, что неравенство $g(t) \leqslant$ $l(t) \leqslant G(t)$ не накладывает на значение $l(t)$ ограничений снизу, а равенство $G(t)=+\infty-$ что на $l(t)$ нет ограничений сверху.

Обычными рассуждениями (см. [6]-[8]) доказьвается

2.0c. УТВЕРЖДЕНИЕ. Два ужа $l$ u $m$ из $L \in \mathrm{TCH}_{n}([a, b])$, порожденных некоторой парой $(g, G)$, не могут иметь первые слева точки своих альтернансов оба на верхней или оба на нижней границе канала $B(g, G)$.

Действительно, в противном случае графики этих ужей имели бы не менее $n$ точек пересечения (с учетом четности нулей разности $l-m$ в этих точках), и мы бы получили $l \equiv m$.

Из утверждения 2.0 с следует

2.0d. УТВЕРЖДЕНИЕ. Каждая пара $(g, G)$ nорождает в $L \in \operatorname{TCH}_{n}([a, b])$ не более двух ужей. Если при этом $L$ содержит уникальньй уж, то он является единственным ужсм в $L$, порожденным парой $(g, G)$ (на самом деле тогда этот уж является единственным әлементом множества $L(g, G)$ см. ниже теорему 2.1).

Теперь чебышевский критерий элемента наилучшего равномерного приближения для $f \in C(\Delta=[a, b])$ и $L \in \mathrm{TCH}_{n}(\Delta)$ можно сформулировать так.

2.0е. Для того чтобы элемент $l$ из $L \in \operatorname{TCH}_{n}(\Delta=[a, b])$ был элементом наилучшего равномерного приближения функиии $f \in C(\Delta)$ әлементами подпространства L, необходимо и достаточно, чтобы l был уникальным ужом из $L$, порожсденным парой $(f-\|l-f\|, f+\|l-f\|)$.

Теорема Карлина [5] утверждает, что если функции $g(x)<G(x)$ определены и непрерывны на отрезке $\Delta$ и сушествует такой элемент $l$ из $L \in \operatorname{TCH}_{n}(\Delta)$, что $g(x)<l(x)<G(x)$ на $\Delta$, то в $L$ имеются ровно два ужа, порожденных парой $(g, G)$.

Следующая теорема исчерпывает обший случай (в [9] она доказана другим, неконструктивным способом).

2.1. ТЕОРемА. Пусть функиии $g$ и $G$ определены на некотором отрезке $\Delta$, $L \in \mathrm{TCH}_{n}(\Delta)$. Тогда при $L(g, G) \neq \varnothing$ имеет место один и только один из двух случаев:

а) $L(g, G)$ состоит из единственного әлемента (әтот случай имеет место тогда и только тогда, когда $L$ содержит уникальный уж, порожденный парой $(g, G))$;

b) $L(g, G)$ состоит более чем из одного элемента; тогда имеются такuе $w \in L u \varepsilon=$ const $>0$, чmо $g(x)+\varepsilon<w(x)<G(x)-\varepsilon$ на $\Delta$ 
(в этом случае необходимым и достаточным условием для наличия в $L$ ровно двух ужей, порожденных парой $(g, G)$, является конечность $W(p, L)$ при $p=(1 /(w-g), 1 /(G-w))$, где $w-$ какой-либо әлемент из $L$, для которого при некотором $\varepsilon>0$ выполнено указанное неравенство).

ДокАЗАТЕЛЬство. 1. Если имеется элемент $l \in L(g, G)$, альтернирующий не более $n$ раз, то возьмем элемент $m \in L$, положительный во всех тех $x \in \Delta$, в которых $l(x)=G(x)$, и отрицательный во всех тех $x \in \Delta$, в которых $l(x)=g(x)$. Тогда

$$
g(x)<w(x):=l(x)-\operatorname{tm}(x)<G(x)
$$

при достаточно малых $t>0$ и всех $x \in \Delta$, т.е. найдутся те $w$ и $\varepsilon$, о которых говорится в п. b) теоремы. Это невозможно в случае а), так что в случае а) единственный в $L(g, G)$ элемент альтернирует не менее $n+1$ раз, т.е. является избыточным ужом. С другой стороны, если имеется избыточный уж $l \in L$, то он является единственным элементом в $L(g, G)$, так как график любого $f \in L(g, G)$ должен пересечься с графиком $l$ не менее $n=\operatorname{dim} L$ раз (с учетом четности - см. [6]), так что $f \equiv l$.

2. Рассматриваем случай b). По сказанному в этом случае в $L(g, G)$ нет избыточных ужей, но есть такие $w \in L$ и $\varepsilon>0$, что при всех $x \in \Delta$ выполняется неравенство $g(x)+\varepsilon<w(x)<G(x)-\varepsilon$. Рассмотрев пару $(g-w, G-w)$, сведем дело к таким функциям $g$ и $G$, что $g(x)+\varepsilon<0<G(x)-\varepsilon$. Считая $g$ и $G$ именно такими, приступаем к рассмотрению случая b).

Необходимость. Пусть существуют два ужа $-f \neq h$, порожденных парой $(g, G)$, и их альтернансы занумерованы в конечные возрастаюшие последовательности $\left\{x_{k}\right\}$ и $\left\{t_{k}\right\}$ соответственно $(k=1, \ldots, n)$. Как уже отмечалось, $f\left(x_{1}\right)$ и $h\left(t_{1}\right)$ имеют разные знаки, для определенности пусть $f\left(x_{1}\right)>0, h\left(t_{1}\right)<0$. Возможны два случая.

$1^{\circ} .\left\{x_{k}\right\}=\left\{t_{k}\right\}$. Тогда $G\left(x_{k}\right)=f\left(x_{k}\right)<\infty$ и $g\left(x_{k}\right)=h\left(x_{k}\right)>-\infty$ при нечетных $k, g\left(x_{k}\right)=f\left(x_{k}\right)>-\infty$ и $G\left(x_{k}\right)=h\left(x_{k}\right)<\infty$ при четных $k$. Положим

$$
r\left(x_{k}\right)=\left(-\frac{1}{g\left(x_{k}\right)}, \frac{1}{G\left(x_{k}\right)}\right), \quad r(x)=(0,0) \quad \forall x \neq x_{k} \quad(x \in \Delta) .
$$

По теореме $0.1 W(r, L)<\infty$, что дает неравенство $W(p, L)<\infty$.

$2^{\circ} .\left\{x_{k}\right\} \neq\left\{t_{k}\right\}$. Пусть $N$ - наименьшее из чисел $k \leqslant n$, для которых $x_{k} \neq t_{k}$ $(1 \leqslant N \leqslant n)$, и, для определенности, $x_{N}<t_{N}$. Тогда для набора из $n+1$ точек $x_{1}<\ldots<x_{N}<t_{N}<t_{N+1}<\ldots<t_{n}$ при нечетном $N$ имеем цепочку из $n+1$ неравенств $G\left(x_{1}\right)<\infty, g\left(x_{2}\right)>-\infty, \ldots, G\left(x_{N}\right)<\infty, g\left(t_{N}\right)>-\infty$, $G\left(t_{N+1}\right)<\infty, g\left(t_{N+2}\right)>-\infty, G\left(t_{N+3}\right)<\infty, \ldots$, а при четном $N$ - цепочку, которая получается из нее, если поменять местами $g$ и $G,>-\infty$ и $<\infty$. И снова из теоремы 0.1 получаем $W(p, L)<\infty$.

Достаточность. Пусть $\Delta=[a, b], \underline{l}$ и $\bar{l}$ - такие элементы из $L$, что $\underline{l}(a)=-1$, $\bar{l}(a)=1, L_{0}=\{l: l \in L, l(a)=0\}, l\left(p, L_{0}, \underline{l}\right)$ и $l\left(p, L_{0}, \bar{l}\right)$ - главные элементы наилучшего приближения соответственно функций $\underline{l}$ и $\bar{l}$ элементами чебышевского на $(a, b]$ подпространства $L_{0}$ с весом $p$. Функции

$$
\lambda:=\frac{l\left(p, L_{0}, \underline{l}\right)-\underline{l}}{E\left(p, L_{0}, \underline{l}\right)}, \quad \mu:=\frac{l\left(p, L_{0}, \bar{l}\right)-\bar{l}}{E\left(p, L_{0}, \bar{l}\right)}
$$


являются искомыми ужами. Действительно, во-первых, при $x \in \Delta$ имеем

$$
\begin{array}{ll}
\left(l\left(p, L_{0}, \underline{l} ; x\right)-\underline{l}(x)\right)^{-}\left(-\frac{1}{g(x)}\right) \leqslant E\left(p, L_{0}, \underline{l}\right), & \lambda(x) \geqslant g(x), \\
\left(l\left(p, L_{0}, \underline{l} ; x\right)-\underline{l}(x)\right)^{+}\left(\frac{1}{G(x)}\right) \leqslant E\left(p, L_{0}, \underline{l}\right), \quad \lambda(x) \leqslant G(x),
\end{array}
$$

т.е. $g(x) \leqslant \lambda(x) \leqslant G(x)$, и, аналогично, имеем $g(x) \leqslant \mu(x) \leqslant G(x)$. Во-вторых (см. введение, п. 0.2), точки $p$-альтернанса пары $\left(l\left(p, L_{0}, \underline{l}\right), \underline{l}\right)$ являются точками альтернанса ужа $\lambda$, пары $\left(l\left(p, L_{0}, \bar{l}\right), \bar{l}\right)$ - точками альтернанса ужа $\mu$.

2.1а. ЗАМЕЧАНИЕ. При $W(p, L)=\infty$ ужей может не быть вовсе (как, например, в случае $\Delta=[0,1], L=\{l(x)=a x+b: a, b \in \mathbb{R}\}$ и пары $(g, G)$, определяемой равенствами $g(0)=-1, G(0)=1, g(x)=-\infty$ и $G(x)=\infty$ при $x \neq 0$ ) или может быть лишь один уж (пример: все, как в предыдушем примере, лишь $G(1)=1$ ).

Следующая теорема - о непрерывной зависимости ужей от канала, т.е. об их устойчивости. Разумеется, вопрос об устойчивости ужа имеет смысл ставить лишь в неособом случае, когда $L(g, G)$ содержит более одного элемента, т.е. в случае b) теоремы 2.1. Так как ужу $l$, порожденному некоторой парой $(g, G)$, при любой функции $w \in L$ взаимно однозначно соответствует уж $l-w$, порожденный парой $(g-w, G-w)$, то, взяв в качестве $w$ какую-либо функцию из п. b) теоремы 2.1 , сводим общий случай к случаю $g(x)<0<G(x)(\forall x \in \Delta)$. При этом несколько упрощается формулировка теоремы.

ОПРЕДЕЛЕНИЕ. Неизбыточные ужи $l, m \in L \in \mathrm{TCH}_{n}(\Delta)$, порожденные парами $(f, F)$ и $(g, G)$ соответственно, называются синхроннымми, если для соответствующих первых слева точек $x_{1}$ и $y_{1}$ альтернансов этих ужей разности $l\left(x_{1}\right)-f\left(x_{1}\right)$ и $m\left(y_{1}\right)-g\left(y_{1}\right)$ равны нулю или положительны одновременно.

2.2. ТЕОРемА (об устойчивости ужей). Пусть $L \in \mathrm{TCH}_{n}(\Delta)$, синхроннье ужи $l$ и т из $L$ порождены парами $(f, F)$ и $(g, G)$ соответственно, $f<0<F, g<0<G$, веса р и q заданы равенствами $p=(-1 / f, 1 / F)$ и $q=(-1 / g, 1 / G), W(p, L)<\infty, W(q, L)<\infty$. Тогда

$$
\|l-m\| \leqslant C \sigma(p, q, L ; d)=C(N \omega(L, d)+d), \quad d=d(p, q),
$$

әде $C=\left\{1 / \underline{s} \cdot W_{2, n}\left(L, \omega^{-1}(2 \underline{s})\right)+1\right\}(W(p, L)+W(q, L)), t=\omega^{-1}(s)$ - обратная $\kappa s=\omega(L, \delta)$ функи, и, $\underline{s}=\min \{S(p /\|p\|, L), S(q /\|q\|, L)\}, \quad N=\max \{\|p\|,\|q\|\}$.

ДокАЗАТЕЛЬСТво. Пусть для определенности ужи $l$ и $m$ получены, как при доказательстве теоремы 2.1 - в результате приближения функции $\underline{l}$ функциями из $L_{0}$ с весами $p$ и $q$, и $E\left(p, L_{0}, \underline{l}\right)=: h \geqslant h^{\prime}:=E\left(q, L_{0}, \underline{l}\right)$. Так как $\|l-\underline{l}\| \geqslant \mid l(a)-$ $\underline{l}(a) \mid=1$ и $\|m-\underline{l}\| \geqslant 1$, то $h \geqslant\|l-\underline{l}\| \cdot S(p, L) \geqslant S(p, L)>0, h^{\prime} \geqslant S(q, L)>0$ и

$$
\begin{aligned}
\|l-m\| & =\left\|\frac{1}{h} \cdot\left(l\left(p, L_{0}, \underline{l}\right)-\underline{l}\right)-\frac{1}{h^{\prime}} \cdot\left(l\left(q, L_{0}, \underline{l}\right)-\underline{l}\right)\right\| \\
& =\left\|\frac{1}{h} \cdot\left(l\left(p, L_{0}, \underline{l}\right)-l\left(q, L_{0}, \underline{l}\right)\right)+\left(\frac{1}{h}-\frac{1}{h^{\prime}}\right)\left(l\left(q, L_{0}, \underline{l}\right)-\underline{l}\right)\right\| \\
& \leqslant \frac{1}{h} \cdot\left\|l\left(p, L_{0}, \underline{l}\right)-l\left(q, L_{0}, \underline{l}\right)\right\|+\left|\frac{1}{h}-\frac{1}{h^{\prime}}\right| \cdot h^{\prime} .
\end{aligned}
$$


Из (1.25.1) имеем

$$
\begin{aligned}
& \frac{1}{h} \cdot\left\|l\left(p, L_{0}, \underline{l}\right)-l\left(q, L_{0}, \underline{l}\right)\right\| \leqslant A \cdot \frac{1}{h} \cdot \sigma\left(p, q, L_{0}(\underline{l}) ; d\right), \\
& \frac{A}{h}=\frac{1}{\underline{s}} \cdot W_{2, n}\left(L_{0}, \omega^{-1}(2 \underline{s})\right)\left(W\left(p, L_{0}(\underline{l})\right)+\frac{h^{\prime}}{h} \cdot W\left(q, L_{0}(\underline{l})\right)\right), \\
& \underline{s}=\min \left\{S\left(\frac{p}{\|p\|}, L_{0}(\underline{l})\right), S\left(\frac{q}{\|q\|}, L_{0}(\underline{l})\right)\right\}, \quad N=\max \{\|p\|,\|q\|\} .
\end{aligned}
$$

Так как $h^{\prime} \leqslant h$, то $\left|h^{-1}-h^{\prime-1}\right| h^{\prime}=\left|h^{\prime}-h\right| / h \leqslant k\left(h^{\prime}, h\right)(k(\cdot, \cdot)$ - обобшенная расстройка - см. введение), и из неравенства (1.5.2) и включения $L_{0}(\underline{l}) \subset L$ получаем

$$
h^{\prime}\left|h^{-1}-h^{\prime-1}\right| \leqslant(W(p, L)+W(q, L)) \cdot \sigma(p, q, L ; d) .
$$

Из (2.2.2)-(2.2.4) следует (2.2.1) $\left(h^{\prime} \leqslant h, L_{0}(\underline{l}) \subset L\right)$.

Доказанное неравенство (2.2.1) симметрично зависит от пар $(f, F)$ и $(g, G)$. Подобно тому, как это мы делали выше, из (2.2.1), учитывая монотонность функции $(1 / t) \cdot W_{2, n}(L, t)$, с помощью теоремы 0.4 легко получить следующие оценки, в которых отправной является пара $(f, F)$.

2.3. СЛЕДСТВИЕ. Если в условиях теоремь 2.2 заменить условие $W(q, L)<\infty$ более сильным неравенством $\|p\| \omega(L, d)+d \leqslant k S(p, L)$, әде $0<k=$ const $<1$, то получим неравенство

$$
\|l-m\| \leqslant C_{1} W(p, L)((1+k)\|p\| \omega(L, d)+d), \quad d=d(p, q)
$$

әде $C_{1}$ не зависит от $q u d$,

$$
C_{1}=\frac{2-k}{1-k} \cdot\left\{\frac{1}{s} \cdot W_{2, n}\left(L, \omega^{-1}(2 s)\right)+1\right\}, \quad s=\frac{1-k}{1+k} \cdot S\left(\frac{p}{\|p\|}, L\right)
$$

Действительно, так как $\|p\| \omega(L, d)+d \leqslant k S(p, L) \leqslant k\|p\|$ (см. введение), то $d \leqslant k\|p\|$. Из неравенства $\|q\| \leqslant\|p\|+d \leqslant\|p\|(1+k)$ и из теоремы 0.4 получаем

$$
\begin{aligned}
S\left(\frac{q}{\|q\|}, L\right) & \geqslant \frac{1}{\|q\|}(S(p, L)-(\|p\| \omega(L, d)+d)) \\
& \geqslant \frac{\|p\|}{\|q\|}(1-k) S\left(\frac{p}{\|p\|}, L\right) \geqslant \frac{1-k}{1+k} S\left(\frac{p}{\|p\|}, L\right), \\
\underline{s} & :=\min \left\{S\left(\frac{p}{\|p\|}, L\right), S\left(\frac{q}{\|q\|}, L\right)\right\} \geqslant \frac{1-k}{1+k} S\left(\frac{p}{\|p\|}, L\right)=: s, \\
W(p, L)+W(q, L) & \leqslant W(p, L)+(S(p, L)-\underline{\sigma}(p, q, L ; d))^{-1} \leqslant \frac{1-k}{1+k} W(p, L),
\end{aligned}
$$

и (2.3.1) следует из (2.2.1). 


\section{§ 2. Примеры ужей, приближение точечного всплеска и точечного колебания в хаусдорфовой метрике}

2.4. Сначала рассмотрим работу одного физического устройства. Пусть имеется некоторая дифференцируюшая $\mathrm{RC}$-цепочка с постоянной времени $\tau=\mathrm{RC}$. Если на ее вход подать короткий положительный П-образный импульс величины $M$ длины $\Delta t$, электрического напряжения $U_{\text {in }}(t)$ то на ее выходе появится знакопеременный импульс $U_{\text {aut }}(t)$, при малых значениях $\tau=\mathrm{RC}$ практически достигающий максимального значения $M$ и минимального $-M$. Чем меньше $\Delta t$, тем граф̆ик входного сигнала $U_{\text {in }}(t)$ визуально (т.е. в

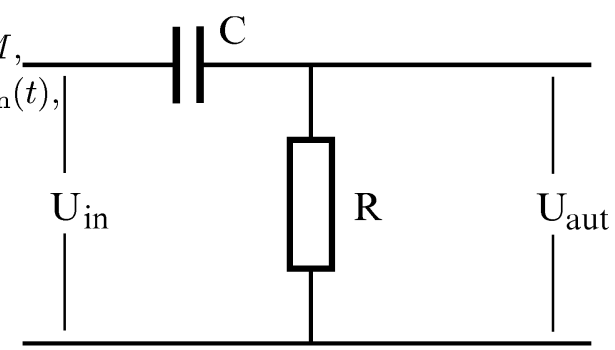
метрике Хаусдорфова - см. введение) все более похож на график многозначной функции $\delta_{M}(t)$, равной $[0, M]$ при $t=0$ и 0 при $t \neq 0$. А чем меньше $\Delta t$ и $\tau / \Delta t$, тем график выходного сигнала $U_{\text {aut }}(t)$ все более похож на график функции $\chi_{M}(t)$, равной $[-M, M]$ при $t=0$ и 0 при $t \neq 0$. Будем назьвать многозначные функции $\delta_{M}(t)$ и $\chi_{M}(t)$ соответственно точечным всплеском величины $M$ и точечнымм колебанием с амплитудой $M$. Если достаточно узкий импульс сигнала $U_{\mathrm{in}}(t)$ и соответствуюший импульс сигнала $U_{\text {aut }}(t)$ рассматривать на экране электронного осциллографа, то (вследствие ограниченности частотной полосы пропускания усилителя вертикального отклонения любого осциллографа) мы увидим вместо графиков импульсов $U_{\text {in }}(t)$ и $U_{\text {aut }}(t)$ графики некоторых бесконечно дифференцируемых функций, визуально близкие к рассматриваемым графикам. При этом если импульсы $U_{\text {in }}(t)$ следуют с постоянной частотой $\nu$, то этими бесконечно дифференцируемыми функциями фактически будут тригонометрические полиномы вида

$$
c_{0}+c_{1} \cos \left(2 \pi \nu t+\alpha_{1}\right)+c_{2} \cos \left(2 \cdot 2 \pi \nu t+\alpha_{2}\right)+\ldots+c_{n} \cos \left(n \cdot 2 \pi \nu+\alpha_{n}\right),
$$

где натуральное число $n$ таково, что гармонические сигналы частот $>n \nu$ уже практически не пропускаются усилителем вертикального отклонения нашего осциллографа. В результате картина импульса, в частности, точечного всплеска или точечного колебания, исказится (свою роль здесь сыграет и явление Гиббса). Естественно возникает вопрос о том, как поступить, чтобы эти искажения были минимальными при пользовании имеющимся осциллографом. Ясно, что если усилитель вертикального отклонения не имеет амплитудно-частотных искажений в полосе частот $[0, n \nu]$, а сигналы с частотами $\geqslant(n+1) \nu$ практически уже не пропускает, то наименьшие искажения периодического импульса с частотой $\nu$ мы получим на экране осциллографа, если на его вход подать не этот импульс, а указанного вида его тригонометрический полином наилучшего приближения в метрике Хаусдорфа. Таким образом, естественно возникает задача о наилучшем хаусдорфовом приближении $2 \pi$-периодических функций тригонометрическими полиномами, в частности, задача о таком приближении $2 \pi$-периодического точечного всплеска $\delta_{M}^{T}(x)$, равного $[0, M]$ при $x=2 \pi k$ и 0 при $x \neq 2 \pi k(k=0, \pm 1, \ldots)$, а также 
$2 \pi$-периодического точечного колебания $\chi_{M}^{T}(x)$, равного $[-M, M]$ при $x=2 \pi k$ и 0 при $x \neq 2 \pi k(k=0, \pm 1, \ldots)$, и задача о наилучших хаусдорфовых приближениях непериодических функций, в частности функций $\delta_{M}(x)$ и $\chi_{M}(x)$, алгебраическими полиномами на некотором отрезке $[a, b] \ni 0$, в частности на $[-1,1]$.

Эти задачи тесно связаны с рассмотренными выше аспектами теории ужей и, более общо, с теорией знакочувствительных аппроксимаций. Задачи о полиномиальных хаусдорфовых приближениях точечного всплеска и точечного колебания интересны еше и тем, что они решаются до конща, и их решения выписываются в явном виде, и что на примере этих простейших многозначных функций в значительной степени проявляется специфика хаусдорфовых аппроксимаций вообше.

Покажем, что задачу о наилучшем хаусдорфовом приближении функций $\delta_{M}(x)$ и $\chi_{M}(x)$ алгебраическими полиномами степени $\leqslant n$ решает (при определенном выборе числа $\delta \in(0,1))$ алгебраический уж $A_{n}(\delta ; x)$, порожденный парой $(g, G)$, где $g(x)=-1$ и $G(x)=1$ при $x \in \sigma(\delta)=[-1,-\delta] \cup[\delta, 1], g(x)=-\infty$ и $G(x)=+\infty$ при $x \notin \sigma(\delta)$, с положительным коэффициентом при $x^{n}$. Очевидно, $A_{n}(0 ; x)=T_{n}(x)=\cos (n \arccos x)$. Вторым ужом $n$-го порядка, порожденньм рассматриваемой парой $(g, G)$, является $-A_{n}(\delta ; x)$. Интересно, что при четном $n$ полином $A_{n}(\delta ; x)$ решает задачу о наилучшем хаусдорфовом приближении функции $\delta_{M}$, а при нечетном - функции $\chi_{M}$.

Перечисленные ниже, в лемме 2.5 , свойства полиномов $A_{n}(\delta ; x)$ непосредственно вытекают из их определения как алгебраических ужей степени $n$, из их единственности, из четности функций $g(x)$ и $G(x)$, из равенства $g(x)=-G(x)$.

2.5. Лемма. а) $A_{1}(\delta ; x) \equiv T_{1}(x) \equiv x$ при любом $\delta \in(0,1)$.

b) При $k \geqslant 1$ и $\delta \in(0,1)$ полином $A_{2 k}(\delta ; x)$ четен, на каждом из отрезков $[-1,-\delta] u[\delta, 1]$ он попеременно $k+1$ раз достигает значений $-1 u$, $\left|A_{2 k}(\delta ; x)\right| \leqslant 1$ при $\delta \leqslant|x| \leqslant 1, A_{2 k}(\delta ; \pm 1)=1, A_{2 k}(\delta ; \pm \delta)=(-1)^{k}$. На отрезке $[-\delta, 0]$ полином $(-1)^{k} A_{2 k}(\delta ; x)$ возрастает от 1 до своего максимального значения $M_{2 k}(\delta)=(-1)^{k} A_{2 k}(\delta ; 0) \quad(>1)$, а на отрезке $[0, \delta]$ убивает от $M_{2 k}(\delta)$ до 1.

с) При $k \geqslant 2$ и $\delta \in[0, \sin (\pi /(4 k-2))]$ полином $A_{2 k-1}(\delta ; x)$ тождественно совпадает с полиномом Чебышева $T_{2 k-1}(x)($ точки $\pm \sin (\pi /(4 k-2))$ - ближайшие к точке $x=0$ әкстремумы полинома Чебышева $\left.T_{2 k-1}(x)\right)$.

d) Если же $\delta \in(\sin (\pi /(4 k-2)), 1)$, то полином $A_{2 k-1}(\delta ; x)$ нечетен, на каждом из отрезков $[-1,-\delta] u[\delta, 1]$ он попеременно $k$ раз достигает значений $-1 u+1,\left|A_{2 k-1}(\delta ; x)\right| \leqslant 1$ npu $\delta \leqslant|x| \leqslant 1, A_{2 k-1}(\delta ; \pm 1)= \pm 1, A_{2 k-1}(\delta ; \pm \delta)=$ $\pm(-1)^{k-1}$. На отрезке $[-\delta, \delta]$ в әтом случае полином $(-1)^{k} A_{2 k-1}(\delta ; x)$ имеет три участка монотонности: вначале он возрастает от 1 до своего максимального значения $M_{2 k-1}(\delta):=\max \left\{(-1)^{k} A_{2 k-1}(\delta ; x): x \in[-1,1]\right\}$ $(>1)$, затем убьвает от $M_{2 k-1}(\delta)$ до $-M_{2 k-1}(\delta)$ и, наконеи, возрастает от $-M_{2 k-1}(\delta) \partial o-1$.

2.6. Нетрудно видеть (сравните с (2.1.1)), что

$$
A_{n}(\delta ; x)=\frac{x^{n}+l\left(p, L_{n-1}, f ; x\right)}{E(p, L, f)}
$$


где $f(x) \equiv-x^{n}, L_{n-1}-$ пространство алгебраических полиномов степени $\leqslant n-1$, $p=\left(p_{-}, p_{+}\right), p_{-}(x)=p_{+}(x)=1 / G(x)$. Таким образом, задача о нахождении явного выражения для $A_{n}(\delta ; x)$ эквивалентна известной задаче о полиномах степени $n$ с равным 1 старшим коэффициентом, наименее отклоняющихся от 0 на множестве $[-1,-\delta] \cup[\delta, 1]$. Последняя задача для полиномов $A_{2 k}(\delta ; x)(k=1,2, \ldots)$ решается элементарно (см. [10, гл. Х, § 52]):

$$
A_{2 k}(\delta ; x) \equiv T_{k}\left(\frac{2 x^{2}-1-\delta^{2}}{1-\delta^{2}}\right) .
$$

Гораздо более сложный вид имеет аналитическое выражение для $A_{n}(\delta ; x)$ при нечетном $n=2 k-1 \quad(k \geqslant 2)$ (по этому поводу см. [10]). Впрочем, случай $\delta \in[0, \sin (\pi /(4 k-2))$ никаких трудностей не представляет: в этом случае $A_{2 k-1}(\delta ; x) \equiv T_{2 k-1}(x)$, так как $T_{2 k-1}$ имеет нужный альтернанс (см. п. с) леммы 2.5).

Из теоремы 2.2 об устойчивости ужей и из некоторых известных общих свойств алгебраических полиномов получается

2.7. ЛЕммА. При $k=1,2, \ldots$ функи ия

$$
M_{n}(\delta):=\max \left\{(-1)^{k} A_{n}(\delta ; x): x \in[-\delta, \delta]\right\}, \quad \delta \in[0,1]
$$

непрерывно и монотонно возрастает, отображая промежуток $[0,1)$ на луч $[1,+\infty)$ при $n=2 k$ или на луч $[0,+\infty)$ при $n=2 k-1$.

При фиксированных $M>0$ и $n=2,3, \ldots$ через $\delta(n)=\delta(n, M)$ обозначим корень уравнения $\delta M_{n}(\delta)+\delta=M$ :

$$
\delta(n) \cdot M_{n}(\delta(n))+\delta(n)=M, \quad \delta(n)=\delta(n, M) \in(0,1)
$$

Существование и единственность $\delta(n)$ на $(0,1)$ непосредственно следует из леммы 2.7.

2.8. ТЕОРемА. Пусть $\Delta=[-1,1]$, число $\delta(n)$ определено, как в (2.7.2), $n \geqslant 2, Q_{n}(M ; x)=(-1)^{[(n+1) / 2]} \cdot \delta(n) A_{n}(\delta(n) ; x)$. Тогда для точечного всплеска $\delta_{M}$ его полином наилучшего приближения $\mathrm{HP}_{n}\left(\delta_{M}, \Delta ; x\right)$ является единственным, причем

$$
\begin{aligned}
\operatorname{HP}_{2 k+1}\left(\delta_{M}, \Delta ; x\right) & \equiv \operatorname{HP}_{2 k}\left(\delta_{M}, \Delta ; x\right) \equiv Q_{2 k}(M ; x), \\
\mathrm{HE}_{2 k+1}\left(\delta_{M}, \Delta\right) & =\operatorname{HE}_{2 k}\left(\delta_{M}, \Delta\right)=\delta(2 k, M)
\end{aligned}
$$

$(k=1,2, \ldots)$, а для точечного колебания $\chi_{M}$ полином $\mathrm{HP}_{n}\left(\chi_{M}, \Delta ; x\right)$ при каждом $n \geqslant 3$ определен с точностью до знака, причем

$$
\begin{aligned}
\mathrm{HP}_{2 k}\left(\chi_{M}, \Delta ; x\right) & \equiv \operatorname{HP}_{2 k-1}\left(\chi_{M}, \Delta ; x\right) \equiv \pm Q_{2 k-1}(M ; x), \\
\mathrm{HE}_{2 k}\left(\chi_{M}, \Delta\right) & =\mathrm{HE}_{2 k-1}\left(\chi_{M}, \Delta\right)=\delta(2 k-1, M)
\end{aligned}
$$

$(k=2,3, \ldots)$. 
ДокАЗАТЕЛЬСТво. Из свойств полинома $A_{n}(\delta ; x)$, отмеченных в лемме 2.5 , и из равенства $\delta(n) M_{n}(\delta(n))=M-\delta(n)$ (см. (2.7.2)) непосредственно следует, что при четном $n$ график полинома $Q_{n}(M ; x)$ и график функции $\delta_{M}(x)$ над отрезком $[-1,1]$ лежат в $\delta(n)$-окрестностях Минковского (см. введение) друг друга, а при нечетном $n$ в $\delta(n)$-окрестностях Минковского друг друга лежат графики функций $y=\chi_{M}(x)$ и $y=Q_{n}(M ; x) \quad(x \in[-1,1])$. Поэтому $H\left(\delta_{M}, Q_{2 k}\right) \leqslant \delta(2 k)$, $H\left(\chi_{M}, Q_{2 k-1}\right) \leqslant \delta(2 k-1)$. Если теперь полином $Q(x)$ степени $\leqslant 2 k+1$ таков, что $H\left(\chi_{M}, Q\right) \leqslant \delta(2 k-1)$, то из очевидных геометрических соображений получаем неравенства

$$
\begin{aligned}
& \max \{Q(x):|x| \leqslant \delta(2 k-1)\} \geqslant M-\delta(2 k-1), \\
& \max \{|Q(x)|: \delta(2 k-1) \leqslant|x| \leqslant 1\} \leqslant \delta(2 k-1) .
\end{aligned}
$$

Отсюда и из того, что $Q_{2 k}(M ; x)$ на каждом из отрезков $[-1,-\delta]$ и $[\delta, 1]$ попеременно $k+1$ раз достигает значений $-\delta(2 k-1)$ и $\delta(2 k-1)$, а при $x=0$ равен

$$
\begin{aligned}
& \max \left\{Q_{2 k}(M ; x):|x| \leqslant \delta(2 k-1)\right\}=\delta(2 k-1) M_{n}(\delta(2 k-1)) \\
& \quad=M-\delta(2 k-1) \leqslant \max \{Q(x):|x| \leqslant \delta(2 k-1)\},
\end{aligned}
$$

следует, что алгебраическое уравнение $Q(x)=Q_{2 k}(M ; x)$ степени $\leqslant 2 k+1$ имеет на отрезке $[-1,1]$ не менее $2 k+2$ корней. Итак, $Q(x) \equiv Q_{2 k}(M ; x)$. Этим доказано, что единственным полиномом наилучшего хаусдорфова приближения степени $\leqslant 2 k+1$, как и степени $\leqslant 2 k$, для функции $\delta_{M}$ является $Q_{2 k}(M ; x)$, и что $\mathrm{HE}_{2 k+1}\left(\delta_{M}, \Delta\right)=\mathrm{HE}_{2 k}\left(\delta_{M}, \Delta\right)=\delta(2 k)$.

Аналогично, если $Q(x)$ - полином степени, не превосходящей $2 k$, для которого $H\left(\chi_{M}, Q\right) \leqslant \delta(2 k-1)$, то либо уравнение $Q(x)=Q_{2 k-1}(M ; x)$, либо уравнение $Q(x)=-Q_{2 k-1}(M ; x)$ имеет на $[-1,1]$ не менее $2 k+1$ корней, так что либо $Q(x) \equiv Q_{2 k-1}(M ; x)$, либо $Q(x) \equiv-Q_{2 k-1}(M ; x)$, откуда следует (2.8.2). Tеорема доказана.

ЗАмечаниЕ. Случаи $n=1$ и $n=2$ для $\operatorname{HP}_{n}\left(\chi_{M}, \Delta ; x\right)$ особые. Так, если $M \geqslant 2$, то полиномы $P_{1}(x)=A x+B$ и $P_{2}(x)=A x+B+C x^{2}$ являются полиномами (степени $\leqslant 1$ и $\leqslant 2$ соответственно) наилучшего хаусдорфова приближения для $\chi_{M}$ при $A$, близких к $M$ или $-M$, и малых $B$ и $C$.

Найдем теперь асимптотику величины $\delta(2 k, M)$ при $k \rightarrow \infty$. Так как $A_{2 k}(\delta ; x) \equiv$ $T_{k}\left(\left(2 x^{2}-1-\delta^{2}\right) /\left(1-\delta^{2}\right)\right)$ (см. (2.6.1)), то уравнение $\delta M_{2 k}(\delta)+\delta=M$ можно записать так:

$$
\delta T_{k}\left(1+\frac{2 \delta^{2}}{1-\delta^{2}}\right)+\delta=M
$$

2.9. ЛЕмма. При каждом $M>0$ решение $\delta(2 k)=\delta(2 k, M)$ уравнения $\delta M_{2 k}(\delta)+\delta=M$ и эквивалентного ему уравнения (2.8.3) имеет при $n=$ $2 k \rightarrow \infty$ следующую асимптотику:

$$
\delta^{*}:=\delta(2 k)=\delta(2 k, M)=\frac{1}{n}\left\{\log (2 M n)-\log \log n+\frac{\log \log n}{\log n}(1+o(1))\right\} .
$$


ДокАЗАТЕЛЬСТво. Поскольку при $x \in \mathbb{R} \backslash[-1,1]$ имеем

$$
T_{k}(x)=\frac{1}{2}\left\{\left(x+\left(x^{2}-1\right)^{1 / 2}\right)^{k}+\left(x-\left(x^{2}-1\right)^{1 / 2}\right)^{k}\right\},
$$

TO

$$
T_{k}\left(1+\frac{2 \delta^{2}}{1-\delta^{2}}\right)=\frac{1}{2}\left\{\left(\frac{1+\delta}{1-\delta}\right)^{k}+\left(\frac{1-\delta}{1+\delta}\right)^{k}\right\} .
$$

Поэтому уравнение $\delta T_{k}\left(1+2 \delta^{2} /\left(1-\delta^{2}\right)\right)+\delta=M$ можно переписать в виде

$$
\delta \cdot \exp \left\{k \cdot \log \frac{1+\delta}{1-\delta}\right\}=2 M+\delta \cdot \gamma(\delta)
$$

где $\gamma(\delta):=-2-(1-\delta)^{k}(1+\delta)^{-k},|\gamma(\delta)| \leqslant 3$, т.е. в виде

$$
\delta \cdot \exp (n \delta) \cdot \exp \left\{n\left(\frac{1}{3} \delta^{3}+\frac{1}{5} \delta^{5}+\ldots\right)\right\}=2 M+\delta \cdot \gamma(\delta) .
$$

Так как при $\delta=(1 / n) \log (2 M n)$ и $n \geqslant 1 / M$ левая часть здесь больше правой, то для корня $\delta(n)$ уравнения $(2.9 .2)$ имеем

$$
\delta(n) \leqslant \frac{1}{n} \log (2 M n) \quad \forall n \geqslant \frac{1}{M} .
$$

Поэтому $\exp \left\{n\left((1 / 3) \delta(n)^{3}+(1 / 5) \delta(n)^{5}+\ldots\right)\right\}=1+O\left(n^{-2} \log ^{3} n\right)(n \rightarrow \infty)$. Отсюда и из (2.9.2) следует, что

$$
\delta(n) \exp \{n \delta(n)\}=2 M+O\left(\frac{\log n}{n}\right), \quad n \rightarrow \infty .
$$

Положим

$$
\delta(n)=: \frac{1}{n} \log \frac{2 M n}{\log n}+\frac{1}{n} r(n)
$$

и оценим $r(n)=r(n, M)$ при $n \rightarrow \infty$. Подставляя выражение (2.9.5) в (2.9.4), получаем

$$
\begin{gathered}
\frac{1}{n \log n}(\log n+\log (2 M)-\log \log n+r(n)) 2 M n \cdot \exp \{r(n)\}=2 M+O\left(\frac{\log n}{n}\right), \\
\left(1+\frac{\log (2 M)}{\log n}-\frac{\log \log n}{\log n}+\frac{r(n)}{\log n}\right) \cdot \exp \{r(n)\}=1+O\left(\frac{\log n}{n}\right) .
\end{gathered}
$$

Отсюда нетрудно усмотреть, что $r(n) \rightarrow 0$ при $n \rightarrow \infty$. В самом деле, если бы для некоторой подпоследовательности $n=n_{s} \rightarrow \infty$ было $r(n)>\varepsilon=\mathrm{const}>0$, то для этих $n$ было бы

$$
\left(1+o(1)+\frac{\varepsilon}{\log n}\right) \cdot \exp \{\varepsilon\} \leqslant 1+O\left(\frac{\log n}{n}\right),
$$


что невозможно при $n \rightarrow \infty$. Аналогично, если бы было $r(n)<-\varepsilon$ при $n=$ $n_{s} \rightarrow \infty$, то (учитывая положительность выражения справа в (2.9.6), а значит, и слева) мы бы имели неравенство

$$
\left(1+o(1)-\frac{\varepsilon}{\log n}\right) \cdot \exp \{-\varepsilon\}>1+O\left(\frac{\log n}{n}\right)
$$

опять же невозможное при $n \rightarrow \infty$. Итак, $r(n) \rightarrow 0$ при $n \rightarrow \infty$, и из (2.9.6) получаем

$$
\left(1-\frac{\log \log n}{\log n}+O\left(\frac{1}{\log n}\right)\right)(1+r(n)(1+o(1)))=1+O\left(\frac{\log n}{n}\right)
$$

откуда

$$
1+r(n)(1+o(1))=1+\frac{\log \log n}{\log n}+O\left(\frac{1}{\log n}\right), \quad r(n)=\frac{\log \log n}{\log n}(1+o(1))
$$

что и требовалось - сравните (2.9.5) и (2.9.1).

Равенство (2.6.1) и лемма 2.9 позволяют дополнить теорему 2.8 следующим утверждением.

2.10. ТЕОРЕМА. При каждом $n=2,3, \ldots$ имеем равенства

$$
\begin{aligned}
& \mathrm{HE}_{n}\left(\delta_{M}, \Delta\right)=\bar{\delta}:=\delta\left(2\left[\frac{n}{2}\right], M\right) \\
& \quad=\frac{1}{n}\left\{\log (2 M n)-\log \log n+\frac{\log \log n}{\log n}(1+o(1))\right\}, \quad n \rightarrow \infty, \\
& \mathrm{HP}_{n}\left(\delta_{M}, \Delta ; x\right)=Q_{2[n / 2]}(M ; x)=(-1)^{[n / 2]} \bar{\delta} T_{[n / 2]}\left(\frac{2 x^{2}-1-\bar{\delta}^{2}}{1-\bar{\delta}^{2}}\right) .
\end{aligned}
$$

Заметим, что в [11] приведен явный вид (2.10.2) для полинома $\mathrm{HP}_{n}\left(\delta_{M}, \Delta ; x\right)$, а также неправильная асимптотика для $\operatorname{HE}_{n}\left(\delta_{M}, \Delta\right): \operatorname{HE}_{n}\left(\delta_{M}, \Delta\right)=(1 / n) \log n+$ $O(1 / n), n \rightarrow \infty$.

Асимптотику величин $\mathrm{HE}_{n}\left(\chi_{M}, \Delta\right)$ можно было бы искать так же, как асимптотику $\mathrm{HE}_{n}\left(\delta_{M}, \Delta\right)$, используя явное выражение для $A_{2 k-1}(\delta ; x)($ см. [10, гл. $\left.\mathrm{X}, \S 52]\right)$. Но при этом возникло бы множество сушественно более серьезных технических трудностей, чем в случае $\delta_{M}$. Поэтому ограничимся более простым результатом, который получается лишш на основании уже установленных выше фактов.

2.11. ТЕОРемА. При каждом $M=$ const $>0$ имеем

$$
\left|\mathrm{HE}_{n}\left(\chi_{M}, \Delta\right)-\frac{1}{n} \log (2 M n)\right| \leqslant \frac{1}{n} \log \log n+o\left(\frac{1}{n}\right), \quad n \rightarrow \infty .
$$


ДокаЗАТЕЛЬСтво. Заметим, что $\mathrm{HE}_{n+1}\left(\chi_{M}\right)>\mathrm{HE}_{n}\left(\delta_{M}\right)$ при $n \geqslant 2$. Действительно, если $Q-$ полином степени $\leqslant 2 k+1$, для которого $H\left(\chi_{M}, Q\right) \leqslant$ $\operatorname{HE}_{2 k}\left(\delta_{M}\right)=\bar{\delta}$, то

$$
\max \{Q(x):|x| \leqslant \bar{\delta}\} \geqslant M-\bar{\delta}, \quad \max \{|Q(x)|: \bar{\delta} \leqslant|x| \leqslant 1\} \leqslant \bar{\delta} .
$$

В доказательстве теоремы 2.8 установлено, что при этих условиях $Q(x) \equiv$ $Q_{2 k}(M ; x) \equiv \operatorname{HP}_{2 k}\left(\delta_{M}, \Delta ; x\right)$. Нетрудно видеть, что замкнутая $\bar{\delta}$-окрестность Минковского точки $(0,-M)$ графика функции $y=\chi_{M}(x)$ не пересекается с графиком функции $y=Q_{2 k}(M ; x)$, что противоречит предположению $H\left(\chi_{M}, Q\right) \leqslant \bar{\delta}$. Итак, $\mathrm{HE}_{2 k+1}\left(\chi_{M}\right)>\mathrm{HE}_{2 k}\left(\delta_{M}\right)$. Вместе с (2.8.1) и (2.8.2) это дает нужное неравенство $\mathrm{HE}_{n+1}\left(\chi_{M}\right)>\mathrm{HE}_{n}\left(\delta_{M}\right)(n \geqslant 2)$. Из него и из (2.10.1) получаем

$$
\operatorname{HE}_{n}\left(\chi_{M}\right)>\frac{\log (2 M n)}{n}-\frac{\log \log n}{n} \quad \forall n \geqslant n_{1}(M),
$$

где $n_{1}(M)$ достаточно велико.

Теперь возьмем $\varepsilon \in(0,1)$ и положим

$$
P(\varepsilon ; x):=\frac{1}{2}\left\{Q_{2 k}\left(\frac{M}{\varepsilon} ; \frac{x-\varepsilon \bar{\delta}}{1+\varepsilon}\right)-Q_{2 k}\left(\frac{M}{\varepsilon} ; \frac{x+\varepsilon \bar{\delta}}{1+\varepsilon}\right)\right\}, \quad \bar{\delta}=\delta\left(2 k, \frac{M}{\varepsilon}\right) .
$$

Очевидно, $P(\varepsilon ; x)$ - нечетный полином степени $\leqslant 2 k-1$. Так как $\left|Q_{2 k}(M / \varepsilon ; t)\right| \leqslant \bar{\delta}$ при $\bar{\delta} \leqslant|t| \leqslant 1$, то

$$
|P(\varepsilon ; x)| \leqslant \bar{\delta} \text { при }(1+2 \varepsilon) \bar{\delta} \leqslant|x| \leqslant 1 .
$$

Оценим снизу величину $M_{\varepsilon}:=\max \{P(\varepsilon ; x):|x| \leqslant(1+2 \varepsilon) \bar{\delta}\}$. Из убывания полинома $Q_{2 k}(x):=Q_{2 k}(M / \varepsilon ; x)$ на $[0, \bar{\delta}]$ следует, что

$$
\begin{aligned}
M_{\varepsilon} & \geqslant \frac{1}{2} \max \left\{Q_{2 k}\left(t-\frac{\varepsilon \bar{\delta}}{1+\varepsilon}\right)-Q_{2 k}\left(t+\frac{\varepsilon \bar{\delta}}{1+\varepsilon}\right): \frac{\varepsilon \bar{\delta}}{1+\varepsilon} \leqslant t \leqslant \frac{\bar{\delta}}{1+\varepsilon}\right\} \\
& =\frac{1}{2} \omega\left(Q_{2 k},[0, \bar{\delta}], \frac{2 \varepsilon \bar{\delta}}{1+\varepsilon}\right)
\end{aligned}
$$

$(\omega(f, E, \delta)$ - модуль непрерывности функции $f$ на множестве $E)$. Так как $\omega(t \delta) \geqslant$ $\omega(\delta) /(1+1 / t)$ для любого $t \in(0,1)$ и любого модуля непрерывности $\omega(\delta)$, то отсюда

$$
\begin{gathered}
M_{\varepsilon} \geqslant \frac{1}{2}\left(\frac{1+\varepsilon}{2 \varepsilon}+1\right)^{-1} \cdot \omega\left(Q_{2 k},[0, \bar{\delta}], \bar{\delta}\right) \\
=\frac{\varepsilon}{1+3 \varepsilon}\left\{Q_{2 k}(0)-Q_{2 k}(\bar{\delta})\right\}=\frac{\varepsilon}{1+3 \varepsilon}\left\{\left(\frac{M}{\varepsilon}-\bar{\delta}\right)-\bar{\delta}\right\} \\
\quad M-2 \varepsilon \bar{\delta} \leqslant(1+3 \varepsilon) M_{\varepsilon} .
\end{gathered}
$$

Рассмотрим нечетный полином $Q(\varepsilon ; x):=(M-2 \varepsilon \bar{\delta}) P(\varepsilon ; x) / M_{\varepsilon}$ степени $\leqslant 2 k-1$. Из (2.11.4) и (2.11.3) при $(1+2 \varepsilon) \bar{\delta} \leqslant|x| \leqslant 1$ имеем

$$
|Q(\varepsilon, x)| \leqslant(1+3 \varepsilon)|P(\varepsilon ; x)| \leqslant(1+3 \varepsilon) \bar{\delta} .
$$


В то же время по определению $M_{\varepsilon}$

$$
\max \{Q(\varepsilon ; x):|x| \leqslant(1+2 \varepsilon) \bar{\delta}\}=M-2 \varepsilon \bar{\delta} .
$$

Отсюда $H\left(\chi_{M}, Q\right) \leqslant(1+3 \varepsilon) \delta(2 k, M / \varepsilon)$, и при $n=2 k-1 \geqslant \varepsilon / M$ из (2.9.3) получаем

$$
\operatorname{HE}_{n}\left(\chi_{M}\right) \leqslant \frac{1}{n}(1+3 \varepsilon) \log \frac{2 M n}{\varepsilon} .
$$

Положив здесь $\varepsilon=1 / \log n$, имеем неравенство

$$
\begin{aligned}
\operatorname{HE}_{n}\left(\chi_{M}\right) & \leqslant \frac{1}{n}\left(1+\frac{3}{\log n}\right)\{\log (2 M n)+\log \log n\} \\
& \leqslant \frac{1}{n}\{\log (2 M n)+\log \log n+4\} \quad \forall n>n_{2}(M)
\end{aligned}
$$

$\left(n_{2}(M)\right.$ достаточно велико). Отсюда и из (2.11.2) следует (2.11.1).

2.12. Вполне аналогично тому, что задачу наилучшего приближения функций $\delta_{M}(x)$ и $\chi_{M}(x)$ алгебраическими полиномами в метрике Хаусдорфа решают алгебраические ужи, задачу о наилучшем хаусдорфовом приближении $2 \pi$-периодических функций $\delta_{M}^{T}(x)$ и $\chi_{M}^{T}(x)$ посредством тригонометрических полиномов решают ужи тригонометрические.

ОПРЕДЕЛЕНИЕ. Пусть на промежутке $\Delta=[a, b)$ задано $n$-мерное чебышевское подпространство $L$ функций $l$, допускаюших непрерывное $(b-a)$-периодическое продолжение, и пара таких функций $g$ и $G$, что $-\infty \leqslant g(x)<G(x) \leqslant+\infty$ $\forall x \in \Delta$. Пусть $F(g)$ и $F(G)$ - дополненные графики $(b-a)$-периодических продолжений $g$ и $G$ соответственно. Тогда элемент $l^{*} \in L$ (и его график $\left.G\left(l^{*}\right)\right)$ назовем $(b-a)$-периодическим ужом из $L$, порожденным парой $(g, G)$, если $g(x) \leqslant$ $l^{*}(x) \leqslant G(x) \forall x$ и на $\Delta$ имеется такой набор из $n-1$ точек $x_{1}<x_{2}<\ldots<x_{n-1}$, что с ростом $k$ от 1 до $n-1$ точки $M_{k}=\left(x_{k}, l^{*}\left(x_{k}\right)\right)$ попеременно попадают то на $F(g)$, то на $F(G)$ (начиная с любого из этих дополненных графиков). Часть графика $G\left(l^{*}\right)$ ужа $l^{*}$ между точками $M_{k} \in F(g)$ и $M_{k+1} \in F(G)$ называется восходящим сегментом ужа $l^{*}$, а между точками $M_{k} \in F(G)$ и $M_{k+1} \in F(g)-$ нисходящим сегментом его (при этом, например, в случае восходяшего сегмента может быть $\left.l^{*}\left(x_{k}\right)>l^{*}\left(x_{k+1}\right)\right)$.

Известно (см. [5], [8]), что если $(b-a)$-периодические функции $g(x)$ и $G(x)$ непрерывны и существует такой элемент $l \in L \in \mathrm{TCH}_{n}(\Delta)$, что $g(x)<l(x)<$ $G(x) \quad \forall x$, то для каждого $t \in \mathbb{R}$ сушествуют ровно два $(b-a)$-периодических ужа $\underline{l}(t ; x)$ и $\bar{l}(t ; x)$ из $L$, порожденных парой $(g, G)$ и проходяших через точку $(t, l(t))$, причем эта точка лежит на нисходяшем сегменте ужа $\underline{l}(t ; x)$ и восходяшем сегменте ужа $\bar{l}(t ; x)$. В общем случае имеет место утверждение, аналогичное теореме 2.1 .

Ниже рассматриваются лишь тригонометрические ужи. При $\lambda \in(0, \pi)$ определим на периоде $[-\pi, \pi] 2 \pi$-периодическую пару $\left(g^{T}, G^{T}\right)$ так: $g^{T}(x)=-1$ и $G^{T}(x)=1$ при $x \in[-\pi,-\lambda] \cup[\lambda, \pi], g^{T}(x)=-\infty$ и $G^{T}(x)=+\infty$ при $x \in(-\lambda, \lambda)$. Пусть $f_{\alpha}(x):=-\cos (n x-\alpha)(\alpha \in \mathbb{R}), p_{-}^{T}(x)=p_{+}^{T}(x)=1 / G^{T}(x), p^{T}=\left(p_{-}^{T}, p_{+}^{T}\right)$, $L_{n-1}^{T}-(2 n-1)$-мерное чебьшевское на $[0,2 \pi)$ пространство всех тригонометрических полиномов порядка $\leqslant n-1$. 
2.13. Нетрудно видеть, что при каждом $\alpha \in \mathbb{R}$ полином

$$
B_{n}(\lambda, \alpha ; x):=\frac{l\left(p^{T}, L_{n-1}^{T}, f_{\alpha} ; x\right)-f_{\alpha}(x)}{E\left(p^{T}, L_{n-1}^{T}, f_{\alpha}\right)}
$$

является ужом порядка $n$, порожденным парой $\left(g^{T}, G^{T}\right)$. Нетрудно также показать, что семейство всех тригонометрических ужей порядка $n$, порожденных парой $\left(g^{T}, G^{T}\right)$, совпадает с семейством $\left\{B_{n}(\lambda, \alpha ; x): \alpha \in \mathbb{R}\right\}$, элементы которого зависят $2 \pi$-периодически и непрерывно от параметра $\alpha$. При этом для каждого фиксированного тригонометрического полинома $T(x)$ порядка $\leqslant n$, для которого $g^{T}(x)<$ $T(x)<G^{T}(x) \forall x$, точки $x_{i}(\alpha)=x_{i}(T ; \alpha)$, в которых $B_{n}\left(\lambda, \alpha ; x_{i}\right)=T\left(x_{i}\right)$, непрерывно и строго монотонно зависят от $\alpha$. Из (2.13.1) получаем тождества

$$
B_{n}(\lambda, \alpha ; x)=-B_{n}(\lambda, \pi+\alpha ; x), \quad B_{n}(\lambda, \alpha ; x)=-B_{n}(\lambda, \pi-\alpha ;-x)
$$

показываюшие, что при описании семейства $\left\{B_{n}(\lambda, \alpha ; x): \alpha \in \mathbb{R}\right\}$ достаточно рассматривать $B_{n}(\lambda, \alpha ; x)$ лишь для $\alpha \in[0, \pi / 2]$. Из (2.13.2) следует четность $B_{n}(\lambda ; x):=B_{n}(\lambda, 0 ; x)$ и нечетность $B_{n}^{*}(\lambda ; x):=B_{n}(\lambda, \pi / 2 ; x)$. Эти полиномы и являются тригонометрическими полиномами порядка $\leqslant n$ наилучшего хаусдорфова приближения для функций $\delta_{M}^{T}(x)$ и $\chi_{M}^{T}(x)$ соответственно.

2.14. Положим

$$
\begin{aligned}
& \mu_{n}(\lambda):=\max \left\{B_{n}(\lambda ; x): x \in[-\lambda ; \lambda]\right\}, \\
& \mu_{n}^{*}(\lambda)=\max \left\{B_{n}^{*}(\lambda ; x): x \in[-\lambda ; \lambda]\right\} .
\end{aligned}
$$

При фиксированных $M>0$ и $n=1,2, \ldots$ корни уравнений

$$
\lambda \mu_{n}(\lambda)+\lambda=M, \quad \lambda \mu_{n}^{*}(\lambda)+\lambda=M
$$

обозначим $\lambda(n)=\lambda(n, M)$ и $\lambda^{*}(n)=\lambda^{*}(n, M)$ соответственно. Сушествование и единственность $\lambda(n)$ и $\lambda^{*}(n)$ на $(0, \pi)$ вытекает из следуюшего утверждения.

2.15. ЛЕмМА. Функиия

$$
M_{n}(\lambda ; \alpha):=\max \{B(\lambda, \alpha ; x): x \in[-\lambda, \lambda]\}
$$

$(0 \leqslant \alpha \leqslant \pi / 2)$ переменного $\lambda \in[0, \pi)$ непрерывна и монотонно возрастает, отображая промежуток $[0, \pi)$ на луч $[\cos \alpha,+\infty)$.

Доказательство этой леммы опирается на устойчивость наименьших уклонений $E\left(p^{T}, L_{n-1}^{T}, f_{\alpha}\right)$ и наилучших приближений $l\left(p^{T}, L_{n-1}^{T}, f_{\alpha} ; x\right)$ при вариациях веса $p^{T}$, в частности вариациях параметра $\lambda$ (см. (2.13.1) и пп. 1.8, 1.17).

Доказательство следуюшего утверждения вполне аналогично доказательству теоремы 2.8 . 
2.16. Теорема. Пусть $n=1,2, \ldots, \quad M>0, \lambda(n) u \lambda^{*}(n)$ - корни первого и второго уравнений (2.14.2) соответственно. Тогда для $2 \pi$-периодического точечного всплеска $\delta_{M}^{T}(x)$ его тригонометрический полином наилучшего хаусдорфова приближения $\mathrm{HT}_{n}\left(\delta_{M}^{T} ; x\right)$ единствен и

$$
\operatorname{HT}_{n}\left(\delta_{M}^{T} ; x\right)=\lambda(n) B_{n}(\lambda(n) ; x), \quad \operatorname{HE}_{n}^{T}\left(\delta_{M}^{T}\right)=\lambda(n),
$$

a для $2 \pi$-периодического точечного колебания $\chi_{M}^{T}(x)$ полином $\operatorname{HT}_{n}\left(\chi_{M}^{T} ; x\right)$ определен с точностью до знака и

$$
\operatorname{HT}_{n}\left(\chi_{M}^{T} ; x\right)= \pm \lambda^{*}(n) B_{n}^{*}\left(\lambda^{*}(n) ; x\right), \quad \operatorname{HE}_{n}^{T}\left(\chi_{M}^{T}\right)=\lambda^{*}(n) .
$$

Нетрудно найти аналитическое выражение для полиномов $B_{n}(\lambda ; x)$ :

$$
B_{n}(\lambda ; x)=T_{n}\left(\frac{2 \cos x+1-\cos \lambda}{1+\cos \lambda}\right) .
$$

Действительно, ввиду (2.6.1) функция

$$
\begin{aligned}
& (-1)^{n} A_{2 n}(\sin (\lambda / 2) ; \sin (x / 2)) \\
& \quad=(-1)^{n} T_{n}\left\{\frac{2 \sin ^{2}(x / 2)-1-\sin ^{2}(\lambda / 2)}{1-\sin ^{2}(\lambda / 2)}\right\}=T_{n}\left\{\frac{2 \cos x+1-\cos \lambda}{1+\cos \lambda}\right\}
\end{aligned}
$$

является четным тригонометрическим полиномом порядка $n$. По п. b) леммы 2.5 и определению тригонометрического ужа порядка $n$ она совпадает с $B_{n}(\lambda ; x)$.

По поводу аналитического выражения для (нечетных) полиномов $B_{n}^{*}(\lambda ; x)$ $(\lambda>\pi /(2 n))$ см. [12].

Теорему 2.16 дополняет

2.17. Теорема. При фиксированных $M>0$ u $n=1,2, \ldots$ тригонометрический полином наилучшего хаусдорфова приближения $\mathrm{HT}_{n}\left(\delta_{M}^{T} ; x\right)$ функции $\delta_{M}^{T}$ является единственным,

$$
\operatorname{HT}_{n}\left(\delta_{M}^{T} ; x\right)=\bar{\lambda} T_{n}\left(\frac{2 \cos x+1-\cos \bar{\lambda}}{1+\cos \bar{\lambda}}\right)
$$

где $T_{n}(x)=\cos (n \arccos x)-$ полином Чебышева $n$-й степени, $\bar{\lambda}=\lambda(n, M)-$ корень уравнения

$$
\lambda T_{n}\left(\frac{3-\cos \lambda}{1+\cos \lambda}\right)+\lambda=M
$$

При $n \rightarrow \infty$ имеет место асимптотическая формула

$$
\begin{aligned}
\mathrm{HE}_{n}^{T}\left(\chi_{M}^{T}\right) & =\bar{\lambda}=\lambda(n, M) \\
& =\frac{1}{n}\left\{\log (2 M n)-\log \log n+\frac{\log \log n}{\log n}(1+o(1))\right\} .
\end{aligned}
$$


ДокАЗАТЕЛЬСТво. Формула (2.17.1) и равенство $\operatorname{HE}_{n}^{T}\left(\chi_{M}^{T}\right)=\bar{\lambda}$ следуют из $(2.16 .1)$ и (2.16.3). Далее заметим, что корень $\delta(n, M)$ уравнения (2.17.2) связан с $\lambda(n, M)$ неравенствами

$$
\begin{aligned}
2 \delta\left(2 n, \frac{M}{2}\right) & \leqslant \lambda(n, M) \leqslant 2 \arcsin \delta\left(2 n, \frac{M}{2}\right) \\
& =2 \delta\left(2 n, \frac{M}{2}\right)+O\left(\delta^{2}\left(2 n, \frac{M}{2}\right)\right) .
\end{aligned}
$$

Действительно, при преобразовании $x=\sin (t / 2)$ четный тригонометрический полином $\operatorname{HT}_{n}\left(\delta_{M}^{T} ; t\right)$ переходит в четный алгебраический полином $P(x)$ степени $2 n$, а четный алгебраический полином $\mathrm{HP}_{2 n}\left(\delta_{M / 2}, \Delta ; x\right)(\Delta=[-1,1])$ - в четньй тригонометрический полином $B(t)$ порядка $n$. При этом преобразовании отрезок $\{t:-\bar{\lambda} \leqslant t \leqslant \bar{\lambda}\}$ соответствует отрезку $\{x:-\sin (\bar{\lambda} / 2) \leqslant x \leqslant \sin (\bar{\lambda} / 2)\}$, а множество $[-\pi,-\bar{\lambda}] \cup[\bar{\lambda}, \pi]$, на котором $\left|\mathrm{HT}_{n}\left(\delta_{M}^{T} ; t\right)\right| \leqslant \bar{\lambda},-$ множеству $[-1,-\sin (\bar{\lambda} / 2)] \cup$ $[\sin (\bar{\lambda} / 2), 1]$, на котором $|P(x)| \leqslant \bar{\lambda}$. Так как

$$
M-\bar{\lambda}=\max \left\{\left|\operatorname{HT}_{n}\left(\delta_{M}^{T} ; t\right)\right|:|t| \leqslant \bar{\lambda}\right\}=\max \left\{|P(x)|:|x| \leqslant \sin \left(\frac{\bar{\lambda}}{2}\right)\right\}
$$

Tо

$$
H\left(\frac{P}{2}, \delta_{M / 2}\right) \leqslant \max \left\{\frac{\bar{\lambda}}{2}, \sin \left(\frac{\bar{\lambda}}{2}\right)\right\}=\frac{\bar{\lambda}}{2}
$$

Аналогично, отрезку $[-\bar{\delta}, \bar{\delta}]$ соответствует отрезок $[-2 \arcsin \bar{\delta}, 2 \arcsin \bar{\delta}]$ и т.д., откуда

$$
H\left(2 B, \delta_{M}^{T}\right) \leqslant \max \left\{2 \delta\left(2 n, \frac{M}{2}\right), 2 \arcsin \delta\left(2 n, \frac{M}{2}\right)\right\}=2 \arcsin \delta\left(2 n, \frac{M}{2}\right) .
$$

Отсюда и следует (2.17.4). Из (2.17.4) и (2.9.1) получим (2.17.3). Теорема доказана.

В заключение заметим, что ужи $B_{n}(\lambda, \alpha ; x)$ решают и более общую задачу о приближении $2 \pi$-периодической функции $f(M, m ; x)$, равной 0 при $x \neq 2 \pi k$ и равной $[m, M](m \leqslant 0 \leqslant M)$ при $x=2 \pi k \quad(k=0, \pm 1, \pm 2, \ldots)$. Именно при определенных значениях параметров $\lambda=\bar{\lambda}=\lambda(n, M, m)$ и $\alpha=\bar{\alpha}=\alpha(n, M, m)$ полином $\bar{\lambda} B_{n}(\bar{\lambda}, \bar{\alpha} ; x)$ является тригонометрическим полиномом порядка $n$ наилучшего хаусдорфова приближения для $f(M, m ; x)$. 


\section{Список литературы}

1. Долженко Е. П., Севастьянов Е. А. Аппроксимации со знакочувствительным весом (теоремы существования и единственности) // Изв. РАН. Сер. матем. 1998. Т. 62. № 6. C. $59-102$.

2. Долженко Е. П., Севастьянов Е. А. Знакочувствительные аппроксимации (пространство знакочувствительных весов, жесткость и свобода системы) // Докл. РАН. 1993. Т. 332. №6. С. 686-689.

3. Долженко Е. П., Севастьянов Е. А. Знакочувствительные аппроксимации (вопросы единственности и устойчивости) // Докл. РАН. 1993. Т. 333. № 1. С. 5-7.

4. Долженко E. П., Севастьянов E. A. Метрические пространства полунепрерывных функций // Матем. заметки. 1994. Т. 55. №3. С. 48-58.

5. Karlin S. Representation theorems for positive functions // J. of Math. and Mech. 1963. V. 12. № 4. P. 599-618.

6. Дзядык B. K. Введение в теорию равномерного приближения функций полиномами. М.: Наука, 1977.

7. Крейн М. Г., Нудельман А. А. Проблема моментов Маркова и экстремальные задачи. М.: Наука, 1973.

8. Карлин $C$., Стадден B. Чебышевские системы и их применение в анализе и статистике. М.: Наука, 1976.

9. Долженко Е. П., Севастьянов Е. А. Об определении чебышевских ужей // Вестник Московского ун-та. Матем. Мех. 1994. № 3. С. 49-59.

10. Ахиезер Н. И. Элементы теории эллиптических функций. М.: Наука, 1970.

11. Сендов Б. Хаусдорфовы приближения. София: Изд-во БАН, 1979.

12. Петухов А.П. Об ужах и приближении разрывных функций в метрике Хаусдорфа // Analysis Mathematica. 1975. T. 11. № 1. C. 55-73.

Поступило в редакцию 3.XI.1997 